TU-654

hep-ph/0206026

June, 2002

\title{
Cosmic Density Perturbations from Late-Decaying Scalar Condensations
}

\author{
Takeo Moroi and Tomo Takahashi \\ Department of Physics, Tohoku University, Sendai 980-8578, Japan
}

\begin{abstract}
We study the cosmic density perturbations induced from fluctuation of the amplitude of late-decaying scalar condensations (called $\phi$ ) in the scenario where the scalar field $\phi$ once dominates the universe. In such a scenario, the cosmic microwave background (CMB) radiation originates to decay products of the scalar condensation and hence its anisotropy is affected by the fluctuation of $\phi$. It is shown that the present cosmic density perturbations can be dominantly induced from the primordial fluctuation of $\phi$, not from the fluctuation of the inflaton field. This scenario may change constraints on the source of the density perturbations, like inflation. In addition, a correlated mixture of adiabatic and isocurvature perturbations may arise in such a scenario; possible signals in the CMB power spectrum are discussed. We also show that the simplest scenario of generating the cosmic density perturbations only from the primordial fluctuation of $\phi$ (i.e., so-called "curvaton" scenario) is severely constrained by the current measurements of the CMB angular power spectrum if correlated mixture of the adiabatic and isocurvature perturbations are generated.
\end{abstract}




\section{Introduction}

In the recent years, observation of the cosmic microwave background (CMB) anisotropy has been greatly improved. After the discovery of the CMB anisotropy by COBE [1] at the angular scale $\theta \gtrsim 7^{\circ}$, there have been many efforts to improve the measurements. In particular, recent balloon and ground-based experiments observed the CMB anisotropy at smaller angular scale of $\theta \sim O\left(0.1^{\circ}\right)$ 2, 3, 4, Then, the observation of the CMB anisotropy is expected to be greatly improved by the on-going and future satellite experiments, MAP [5] and PLANCK [6]; after these experiments, the CMB angular power spectrum $C_{l}$ will be determined at $O(1 \%)$ level up to the multipole $l \lesssim 1000-2000$.

With these measurements, our understanding of the evolution of the universe is also being improved. Importantly, since the CMB power spectrum is sensitive to origins and evolutions of the cosmic density perturbations (as well as to the cosmological parameters), we are now able to constrain scenarios of generating the cosmic density perturbations.

Among various scenarios, inflation [7] is probably the most popular and well-motivated one to provide the source of the cosmic density perturbations. In the simplest case, all the components in the universe (other than the cosmological constant), like the photon, baryon, cold dark matter (CDM), and so on, originate to decay products of the inflaton field $\chi$ which is the scalar field responsible for the inflation. The inflaton field fluctuates during the inflation and it becomes the source of the cosmic density perturbations. One of the most important consequences of such a scenario is that, since all the components in the universe are produced from the inflaton field, there is no entropy perturbation between any of two components and hence the density fluctuations become adiabatic. Importantly, assuming the standard evolution of the universe after the inflation and using a reasonable set of the cosmological parameters, it is now widely believed that the observed CMB power spectrum is consistent with the one predicted from the scale-invariant spectrum which can arise from some class of inflation models [8].

From the particle-physics point of view, however, the simplest scenario may not be the case and there exist possible sources of the cosmic density perturbations other than the inflaton field. In particular, in various scenarios, light scalar fields are introduced which dominate the universe at early epochs. (We denote such a scalar field as $\phi$.) For example, in the scenario of Affleck-Dine baryogenesis [9], condensation of the squark and slepton fields is converted to the baryon-number asymmetry of the universe. If this is the case, there may exist an epoch when the universe is dominated by the Affleck-Dine field. Then, at the time of the decay of the Affleck-Dine field, the late-time entropy production occurs. Other candidate is flat directions in the theory space. In particular, in the superstring theory [10], there are various flat directions parameterized by scalar fields called moduli fields. Such moduli fields are expected to acquire masses from the effect of the supersymmetry breaking and hence their masses can be much lighter than the Planck scale. If the initial amplitudes of the moduli fields are large, the universe is once dominated by the moduli field and is reheated at the time of the decay of the moduli fields. (See Refs. [11, 12 for the scenario with cosmological moduli fields.) In addition, in Refs. [13] it is pointed out 
that the baryon-number asymmetry of the universe can be explained if the universe was once dominated by the condensation of the right-handed scalar neutrino. In this case, the universe also experiences the late-time entropy production. In addition, axion-like scalar field is proposed as a seed of the cosmic density perturbations [14 in the pre-big-bang [15] and the ekpyrotic [16] scenarios.

Such scalar fields may acquire fluctuations of the amplitude in the early universe (in particular, during the inflation). Then, the scalar field eventually decays and reheats the universe. Importantly, in this class of scenario, the CMB radiation we observe today originates to $\phi$ rather than to the inflaton. As a result, one can expect that the fluctuation of the scalar-field amplitude affects the cosmic density perturbations [17, 18, 19, 20]. In particular, in such a scenario, adiabatic and isocurvature perturbations may be generated with cross-correlation. Indeed, in Ref. 20], the CMB angular power spectrum is calculated in such a framework, and it was shown that effects of the correlated isocurvature perturbation may be large enough to be seen in the on-going and future experiments. In addition, it is possible that the dominant part of the cosmic density perturbations observed today may originate to the primordial perturbation in the amplitude of $\phi$ rather than to the fluctuation of the inflaton amplitude. (This kind of mechanism is sometimes called "curvaton mechanism.")

In this paper, we consider effects of such scalar-field condensations to the cosmic density perturbations without relying on any particular scenarios. Assuming that the universe was once dominated by the scalar-field condensation and that the reheating occurred at a later stage with generating large amount of entropy, we study the evolutions of the cosmic density perturbations and consider the CMB angular power spectrum. As we will see, the CMB power spectrum can be affected in various ways depending on scenarios. Interestingly, if the decay product of the $\phi$ field does not generate the baryon asymmetry or the CDM component, then the adiabatic and isocurvature perturbations can be both generated with cross-correlation. In this case, the CMB power spectrum may significantly change its behavior from the adiabatic result. In particular, the simple "curvaton" scenario, in which the cosmic density perturbations are generated only from the primordial fluctuation of the amplitude of $\phi$, is severely constrained by the current observations of the $\mathrm{CMB}$ angular power spectrum if the adiabatic and isocurvature perturbations are generated with cross-correlation.

The organization of the rest of this paper is as follows. In Section 2, we introduce the scenario we consider and follow the thermal history of the universe. Evolutions of the cosmic density perturbations in such a scenario is discussed in Section 3. In particular, it is discussed how the density perturbation in the radiation is affected by the primordial fluctuation of the amplitude of $\phi$. Then, in Section 4 , we overview the behavior of the CMB anisotropy in our scenario. Detailed discussions on the CMB power spectrum for the cases with and without the isocurvature perturbations are given in Sections 5 and 6 , respectively. Section 7 is devoted for the conclusion.

\footnotetext{
${ }^{\# 1}$ For other mechanisms of generating correlated mixture of the adiabatic and isocurvature perturbations, see [21].
} 


\section{Scenario}

Let us first introduce the scenario we have in mind. In this paper, we consider the thermal history with late-time entropy production which is due to the decay of the scalar field $\phi$.

For our study, it is important to understand the behavior of the scalar field in the expanding universe. For this purpose, we start with the Lagrangian of the form

$$
\mathcal{L}=\frac{1}{2} g^{\mu \nu} \partial_{\mu} \phi \partial_{\nu} \phi-V(\phi)
$$

where $V(\phi)$ is the potential of the scalar field. In our discussion, we adopt the parabolic potential for the scalar field;

$$
V(\phi)=\frac{1}{2} m_{\phi}^{2} \phi^{2}
$$

From this Lagrangian, we can derive the energy density $\rho_{\phi}$ and the pressure $p_{\phi}$ of the scalar field condensation; for the zero-mode, we obtain

$$
\rho_{\phi}=\frac{1}{2} \dot{\phi}^{2}+\frac{1}{2} m_{\phi}^{2} \phi^{2}, \quad p_{\phi}=\frac{1}{2} \dot{\phi}^{2}-\frac{1}{2} m_{\phi}^{2} \phi^{2},
$$

where the "dot" denotes the derivative with respect to time $t$.

In addition, in the expanding universe, equation of motion of the scalar field is given by

$$
\ddot{\phi}+3 H \dot{\phi}+m_{\phi}^{2} \phi=0
$$

where $H \equiv \dot{a} / a$ is the expansion rate with $a$ being the scale factor. The behavior of $\phi$ depends on the relative size of the expansion rate $H$ and the scalar mass $m_{\phi}$. When the expansion rate is much larger than $m_{\phi}$, the second term, which plays the role of the friction term, is significant and $\dot{\phi}$ (almost) vanishes. In this case, change of the scalar amplitude can be neglected and $\phi$ takes a constant value. We call this epoch as "slow-roll" epoch since $\dot{\phi}$ is negligibly small. On the contrary, when $H \lesssim m_{\phi}$, the scalar field oscillates around the minimum of the potential. In particular, when $H \ll m_{\phi}$, the oscillation of the scalar field becomes fast enough so that the relation $\frac{1}{2}\left\langle\dot{\phi}^{2}\right\rangle_{\text {osc }}=\frac{1}{2} m_{\phi}^{2}\left\langle\phi^{2}\right\rangle_{\text {osc }}$ holds, where $\langle\cdots\rangle_{\text {osc }}$ denotes the average over the time scale much longer than $m_{\phi}^{-1}$ (but shorter than $H^{-1}$ ). With this relation, the energy density and the pressure of the scalar field are given as

$$
\left\langle\rho_{\phi}\right\rangle_{\mathrm{osc}}=\left\langle\frac{1}{2} \dot{\phi}^{2}+\frac{1}{2} m_{\phi}^{2} \phi^{2}\right\rangle_{\mathrm{osc}}=m_{\phi}^{2}\left\langle\phi^{2}\right\rangle_{\mathrm{osc}}, \quad\left\langle p_{\phi}\right\rangle_{\mathrm{osc}}=\left\langle\frac{1}{2} \dot{\phi}^{2}-\frac{1}{2} m_{\phi}^{2} \phi^{2}\right\rangle_{\mathrm{osc}}=0 .
$$

As we can see, the pressure of the scalar field effectively vanishes at this epoch and the equation-of-state parameter for the scalar field becomes $\omega_{\phi}=0$. In this case, the energy density of the scalar field behaves as that of the non-relativistic component. Consequently, when $\Gamma_{\phi} \ll H \ll m_{\phi}$ (with $\Gamma_{\phi}$ being the decay rate of $\phi$ ), $\rho_{\phi}$ decreases as $a^{-3}$. 
Now we discuss the thermal history of the universe with the scalar field $\phi$. In particular, we consider the case where the initial amplitude of the scalar field $\phi_{\text {init }}$ is non-vanishing so that $\phi$ may become cosmologically significant. With such an initial condition, let us follow the evolution of the universe starting with the inflationary period.

As in the conventional cases, we adopt the epoch of (slow-roll) inflation in the early stage. As well as solving the horizon and flatness problems, inflation provides the source of the cosmic density perturbations. We assume that the mass of the scalar field $\phi$ is much smaller than the expansion rate of the universe during the inflation $H_{\mathrm{inf}}$. If this is the case, the energy density of the scalar field is much smaller than the total energy density of the universe as far as the amplitude of the scalar field is smaller than the Planck scale. Then, the potential of the scalar field $\phi$ is effectively flat during inflation and there is no strong reason to have vanishing initial amplitude of $\phi$. In addition, if $m_{\phi} \ll H_{\text {inf }}$, $\phi$ is slow-rolling during inflation and it keeps its initial amplitude. Thus, we assume a non-vanishing value of the initial amplitude $\phi_{\text {init }}$.

In the slow-roll inflation models, the inflaton field $\chi$ oscillates after the inflation and decays. Then, the universe is reheated. (We call this epoch as the "first reheating" since, in the scenarios we consider, the universe is reheated again due to the decay of $\phi$.) The energy density of the inflaton field also decreases as $a^{-3}$ in this oscillating period, and hence the expansion rate of the universe is being decreased. Then, at the time when the expansion rate of the universe becomes comparable to the decay rate of the inflaton $\Gamma_{\chi}$, the inflaton decays and reheats the universe. Equating the expansion rate and the decay rate of the inflaton field, the reheating temperature is estimated to be

$$
T_{\mathrm{R} 1} \sim g_{*}^{-1 / 4} \sqrt{\Gamma_{\chi} M_{*}},
$$

where $g_{*} \sim O(100)$ is the effective number of the massless degrees of freedom, 屙 $M_{*} \simeq$ $2.4 \times 10^{18} \mathrm{GeV}$ is the reduced Planck scale, and we adopt the approximation of the instantaneous reheating at the time of $H=\Gamma_{\chi}$. In addition, here (and in the following order-of-estimations in this section), we neglect numerical constants of $O(1)$.

Evolution of the scalar field $\phi$ depends on the relative size of $m_{\phi}$ and $\Gamma_{\chi}$. If the mass $m_{\phi}$ is smaller than $\Gamma_{\chi}, \phi$ starts its oscillation after the decay of the inflaton; otherwise, $\phi$ starts to move while the inflaton field is still oscillating. In studying the thermal history with the scalar field $\phi$, it is also important to specify the initial value of the scalar field $\phi_{\text {init. }}$. When $\phi$ starts to move, $H \sim O\left(m_{\phi}\right)$ and hence the total energy density of the universe is $O\left(m_{\phi}^{2} M_{*}^{2}\right)$. Thus, if $\phi_{\text {init }}$ is smaller than $M_{*}$, the scalar field is a sub-dominant component when it starts to move, and hence the evolution of the universe at this epoch is controlled by other components, oscillating inflaton field or radiation. If $\phi_{\text {init }} \sim M_{*}$, on the contrary, energy density of $\phi$ becomes comparable to the total energy density. In this case, the scalar field $\phi$ never becomes minor component until it decays.

\footnotetext{
\#2 If the minimum of the potential of $\phi$ is a symmetry-enhanced point, however, initial value of $\phi$ may vanish 22 .

\#3 In this section, we neglect the temperature dependence of $g_{*}$ since it does not significantly change the following discussion.
} 
Let us first consider the case where $T_{\mathrm{R} 1}$ is high enough so that $\phi$ starts to oscillate after the decay of the inflaton. This happens when $m_{\phi} \lesssim \Gamma_{\chi}$. In this case, the scalar field starts to oscillate at the temperature

$$
T_{\mathrm{osc}} \sim g_{*}^{-1 / 4} \sqrt{m_{\phi} M_{*}} .
$$

After this epoch, the energy density of the scalar field $\rho_{\phi}$ decreases as $a^{-3}$ while that of radiation is proportional to $a^{-4}$, and hence the energy density of the scalar field decreases slower than that of the radiation. Thus, when the amplitude of the scalar field is as large as $\phi_{\text {eq }} \sim \phi_{\text {init }}^{4} / M_{*}^{3}$, the energy density of the scalar field becomes comparable to that of radiation if the scalar field does not decay before this epoch. Expansion rate at this epoch is $H_{\text {eq }} \sim\left(\phi_{\text {init }}^{4} / M_{*}^{4}\right) m_{\phi}$, and $H_{\text {eq }}$ becomes larger than the decay rate of $\phi$ if

$$
\phi_{\text {init }} \gtrsim\left(\Gamma_{\phi} / m_{\phi}\right)^{1 / 4} M_{*} \sim g_{*}^{1 / 8}\left(T_{\mathrm{R} 2}^{2} / m_{\phi} M_{*}\right)^{1 / 4} M_{*} \quad: \quad m_{\phi}<\Gamma_{\chi},
$$

where $T_{\mathrm{R} 2}$ is the reheating temperature after the decay of $\phi$ and is estimated as

$$
T_{\mathrm{R} 2} \sim g_{*}^{-1 / 4} \sqrt{\Gamma_{\phi} M_{*}} .
$$

If the condition (2.8) is satisfied, the scalar field dominates the universe before it decays.

If the reheating temperature after the inflation $T_{\mathrm{R} 1}$ becomes low, the scalar field $\phi$ may start to oscillate before the inflaton field decays; this is the case if $\Gamma_{\chi} \lesssim m_{\phi}$. In this case, when the inflaton field decays, the amplitude of the scalar field is $\left(\Gamma_{\chi} / m_{\phi}\right) \phi_{\text {init }}$. Then, the energy densities of $\phi$ and radiation become comparable when $\phi \sim\left(\Gamma_{\chi} / m_{\phi}\right) \phi_{\text {init }}^{4} / M_{*}^{3}$, if $\phi$ does not decay before its amplitude becomes smaller than this value. Then, the condition to have a $\phi$-dominated epoch is

$$
\phi_{\text {init }} \geq\left(\Gamma_{\phi} / \Gamma_{\chi}\right)^{1 / 4} M_{*} \sim\left(T_{\mathrm{R} 2} / T_{\mathrm{R} 1}\right)^{1 / 2} M_{*} \quad: \quad m_{\phi}>\Gamma_{\chi} .
$$

If the initial amplitude of $\phi$ becomes larger than $\sim M_{*}$, on the other hand, the $\phi$ field slow-rolls even after the expansion rate of the universe becomes comparable to $m_{\phi}$. Then, the universe enters into the de Sitter phase again. In this case, $\phi$ plays the role of the inflaton and the resultant cosmic density perturbation becomes the same as the standard adiabatic result (if the scale of the secondary inflation is properly chosen).\#4 Thus, we assume that the initial amplitude of $\phi$ is smaller than $M_{*}$. In particular, we assume that $\phi_{\text {init }}$ is small enough so that the universe has a radiation-dominated epoch before $\phi$ dominates the universe. (This is the case if $\phi_{\text {init }}$ is substantially smaller than $M_{*}$.)

In Fig. 1, we summarize the above results; on the $T_{\mathrm{R} 2}$ vs. $m_{\phi}$ plane, we plot the contours of the minimum value of $\phi_{\text {init }}$ with which the universe experiences the $\phi$-dominated epoch.

\#4 If the $e$-folding number due to the secondary inflation is small, however, this is not the case and the cosmic density fluctuation for large scale is generated by the first inflation. We do not consider such a case in this paper. 


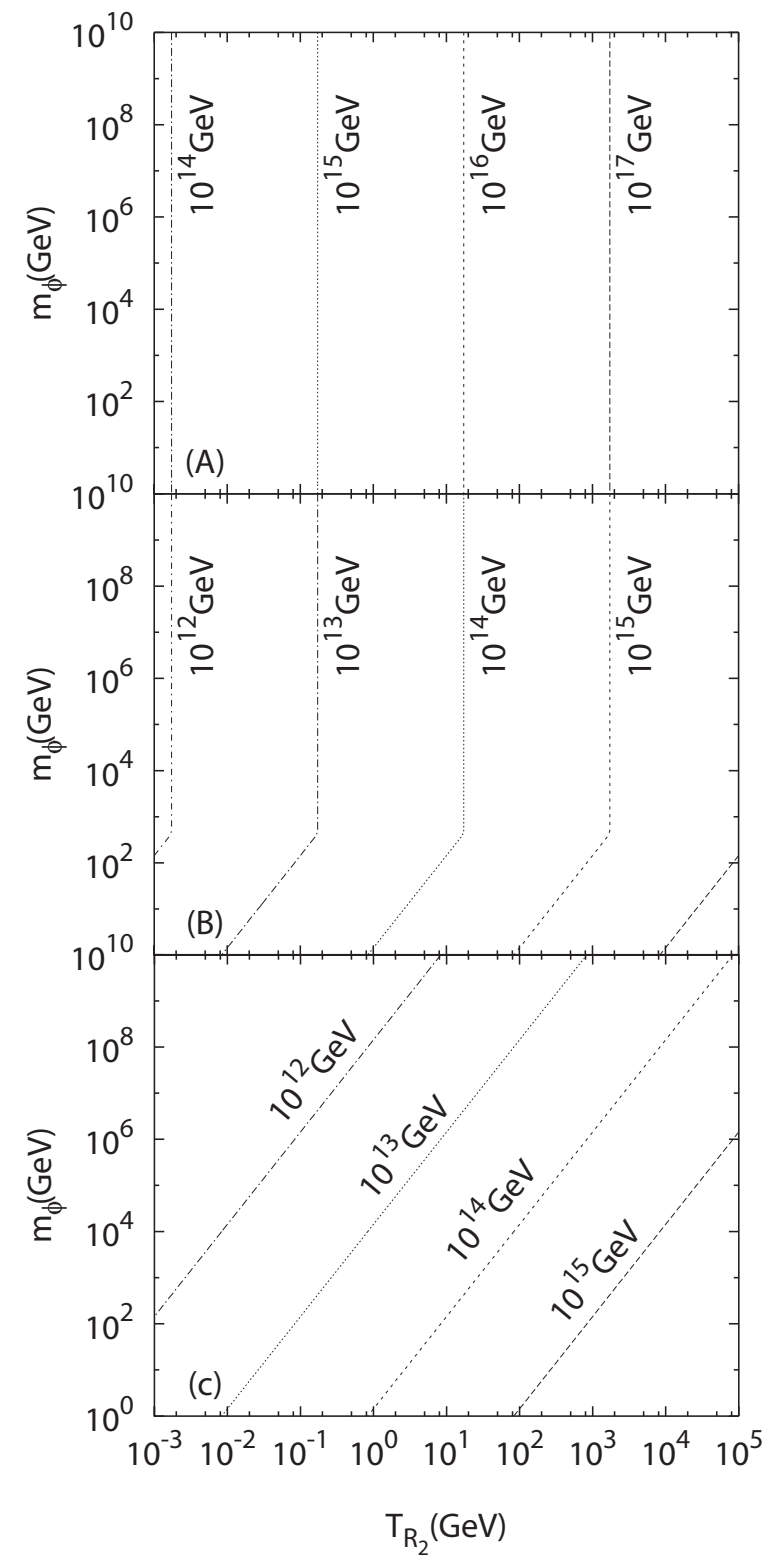

Figure 1: Contours of minimum values of the initial amplitude of the $\phi$ field with which the universe is once dominated by the energy density of $\phi$. The vertical axis is the mass of $\phi$ and the horizontal axis is $T_{\mathrm{R} 2} . T_{\mathrm{R} 1}$ is taken to be (A) $T_{\mathrm{R} 1}=1 \times 10^{6} \mathrm{GeV}$, (B) $T_{\mathrm{R} 1}=1 \times 10^{10} \mathrm{GeV}$ and $(\mathrm{C}) T_{\mathrm{R} 1}=1 \times 10^{14} \mathrm{GeV}$. 
In the following discussion, we assume that the initial amplitude of the scalar field is large enough so that there exists the $\phi$-dominated epoch.

Since the universe has to follow the standard big-bang scenario in particular for the bigbang nucleosynthesis, however, the universe has to become radiation-dominated after the $\phi$-dominated epoch. This is realized by the reheating due to the decay of $\phi$. When $H \sim \Gamma_{\phi}$, the $\phi$ field decays and its energy density is converted to that of radiation. The reheating temperature at the time of the $\phi$-decay depends on the decay rate $\Gamma_{\phi}$. Importantly, the reheating temperature has to be higher than $\sim 1 \mathrm{MeV}$ so that the success of the standard big-bang nucleosynthesis is not altered; we assume that $\Gamma_{\phi}$ is large enough in order not to affect the standard nucleosynthesis scenario.

In summary, the thermal history is as follows:

1. In the early stage, inflation occurs.䛻 During inflation, the scalar field $\phi$ has a substantial initial amplitude so that it dominates the universe at the later stage.

2. After the inflation, the universe is dominated by the oscillating inflaton field. (We call this epoch as " $\chi \mathrm{D}$ " epoch.) At some point, the inflaton decays and reheats the universe.

3. When the expansion rate of the universe becomes comparable to $m_{\phi}$, the $\phi$ field starts to oscillate. This may happen during the $\chi \mathrm{D}$ or the radiation-dominated epoch. We assume that the initial amplitude of $\phi$ is smaller than $\sim M_{*}$ so that there is no inflation induced by the $\phi$ field. In addition, if $\phi_{\text {init }}$ is substantially smaller than $M_{*}$, the universe experiences a radiation-dominated epoch before $\phi$ dominates the universe. (We call this epoch as "RD1" epoch.)

4. As the universe expands, $\phi$ eventually dominates the universe since $\rho_{\phi} \propto a^{-3}$ while $\rho_{\text {rad }} \propto a^{-4}$ (where $\rho_{\text {rad }}$ is the energy density of radiation). We call this epoch " $\phi \mathrm{D}$ " epoch.

5. When $H \sim \Gamma_{\phi}$, the $\phi$ field decays and reheats the universe. Then the radiationdominated universe is realized again. (We call this epoch "RD2" epoch.) After this epoch, the evolution of the universe is the same as the standard big-bang case as far as the cosmic density perturbations are not concerned.

The scenario is schematically shown in Fig. 2. (In the following, the subscripts " $\chi \mathrm{D}$, , "RD1," " $\phi \mathrm{D}, "$ and "RD2" will be used for variables in each epochs.)

\section{Evolutions of the Perturbations}

In the previous section, we summarized the scenario and discussed the evolutions of the background variables (i.e., the zero-modes). Our purpose is, however, to understand the

\footnotetext{
${ }^{\# 5}$ For the pre-big-bang [15] and the ekpyrotic [16] scenarios, this may not be the case. In these cases, we assume that some other mechanism generates the primordial fluctuation of $\phi$.
} 




Figure 2: Schematic picture of the thermal history of the universe; evolution of the energy density of various components. Here, we assumed that the $\phi$ field starts to oscillate in the RD1 epoch, but it may happen in the $\chi \mathrm{D}$ epoch. The solid lines are for scalar condensations and the dotted ones are for radiation.

effects of the fluctuation of the amplitude of the scalar field $\phi$ on the cosmic density perturbations. Thus, in this section, we discuss the evolutions of the perturbations in detail. Importantly, properties of the density perturbations are determined at the epoch much earlier than the electron decoupling (i.e., $T \sim m_{e}$, with $m_{e}$ being the electron mass) and the neutrino freeze-out. Thus, in this section, we study the behaviors of the perturbations in such an epoch. In this paper, we give the formalism in the conformal Newtonian gauge. (We follow the notation and convention of [23].)

In discussing the evolutions of the perturbations, it is convenient to define the variable $\delta_{X}$ as

$$
\delta_{X} \equiv \delta \rho_{X} / \rho_{X},
$$

where the subscript $X$ denotes the individual components (like radiation, CDM, baryon, and so on) and $\delta \rho_{X}$ is the density perturbation of the component $X$. In addition, in the conformal Newtonian gauge, the perturbed line element is given by

$$
\begin{aligned}
d s^{2} & =-(1+2 \Psi) d t^{2}+\left(\frac{a}{a_{0}}\right)^{2}(1+2 \Phi) \delta_{i j} d x^{i} d x^{j} \\
& =\left(\frac{a}{a_{0}}\right)^{2}\left[-(1+2 \Psi) d \tau^{2}+(1+2 \Phi) \delta_{i j} d x^{i} d x^{j}\right],
\end{aligned}
$$


where $\tau$ is the conformal time, $a_{0}$ is the scale factor at the present time, and $\Psi$ and $\Phi$ are the metric perturbations.

Substituting Eq. (3.2) into the Einstein equation, we obtain the generalized Poisson equation for $\Phi$ :

$$
k^{2} \Phi=\frac{1}{2 M_{*}^{2}}\left(\frac{a}{a_{0}}\right)^{2} \rho_{\text {tot }}\left[\delta_{\text {tot }}+\frac{3 \mathcal{H}}{k}\left(1+\omega_{\text {tot }}\right) V_{\text {tot }}\right]
$$

and other equation relating $\Phi, \Psi$, and the anisotropic stress perturbation of the total matter $\Pi_{\text {tot }}$ :

$$
k^{2}(\Psi+\Phi)=-\frac{1}{M_{*}^{2}}\left(\frac{a}{a_{0}}\right)^{2} p_{\text {tot }} \Pi_{\text {tot }} .
$$

Here, "tot" denotes the total matter and the variable $V_{X}$ denotes the velocity perturbation of the component $X$. In addition, $k$ is the comoving momentum, $\omega_{\text {tot }} \equiv \rho_{\text {tot }} / p_{\text {tot }}$ the equation-of-state parameter for the total matter, and

$$
\mathcal{H} \equiv \frac{1}{a} \frac{d a}{d \tau} .
$$

As indicated in the above equations, evolutions of the metric perturbations are related to those of the density, velocity, and other perturbations of various components. Here, we consider the universe with temperature higher than $T \simeq m_{e}$. In such a situation, various charged particles become relativistic and are thermally produced. Then, all the relativistic components (like photon, electron, quarks, neutrinos, and so on) can be regarded as a single fluid with very short mean free path, much shorter than the horizon scale. We call such a component as "radiation" and use the subscript " $r$ " for it.

When the mean free path of the radiation is very short, perturbation of the radiation becomes locally isotropic and the anisotropic stress perturbation vanishes:

$$
\Pi_{r}=0 .
$$

Then, the equations for the density and velocity perturbations of the radiation are given by

$$
\begin{aligned}
\delta_{r}^{\prime} & =-\frac{4}{3} k V_{r}-4 \Phi^{\prime}, \\
V_{r}^{\prime} & =\frac{1}{4} k \delta_{r}+k \Psi,
\end{aligned}
$$

where the "prime" denotes the derivative with respect to the conformal time $\tau$. In addition, if a very weakly interacting non-relativistic component exists, its perturbations obey the following equations:

$$
\begin{aligned}
\delta_{m}^{\prime} & =-k V_{m}-3 \Phi^{\prime}, \\
V_{m}^{\prime} & =-\mathcal{H} V_{m}+k \Psi,
\end{aligned}
$$


where the subscript " $m$ " is for non-relativistic matters. Notice that, when a scalar field is oscillating, the equation-of-state parameter of the scalar condensation vanishes and hence the density and velocity perturbations of the scalar field also obey Eqs. (3.9) and (3.10).

In our scenario, there are several possible sources of the density perturbations. The first one is, as in the conventional case, fluctuation of the inflaton amplitude. In our case, however, amplitude of $\phi$ may also fluctuate and such a fluctuation becomes second source of the density fluctuations. In addition, if there exists independent scalar field (called $\psi$ ) from which the baryon or the CDM is generated, we also have to take account of its effects. (In the following, we consider the cases with and without this scalar field $\psi$.) In the following, primordial fluctuations of these scalar fields are assumed to be uncorrelated. In the framework of the linear perturbation theory, evolutions of the perturbations from the primordial fluctuations of these scalar fields are independent and hence we can study their evolutions separately. Evolutions of the perturbations from the inflaton fluctuation are intensively studied; such a fluctuation provides adiabatic density fluctuations. In addition, density perturbation induced from the fluctuation of $\psi$ is also well known as far as $\psi$ is a minor component in the RD2 epoch; primordial fluctuation of $\psi$ induces (purely) isocurvature fluctuation to the baryonic or the CDM component.

Thus, in the following, we study the evolutions of the density perturbations induced by the fluctuation of the amplitude of $\phi$. For this purpose, we decompose the scalar field as

$$
\phi(t, k)=\phi(t)+\delta \phi(k, t)
$$

where $\delta \phi$ denotes the fluctuation of $\phi$. (We use the same notation for the total amplitude and for the zero-mode because we believe there is no confusion.) In particular we study evolutions in very early universe in order to understand how the fluctuations behave when the universe enters into the RD2 epoch. In realistic scenarios, the scale we are interested in (i.e., the scale corresponding to the multipole $l \lesssim O(1000)$ ) is far outside of the horizon at this epoch and hence we can expand the solutions to the equations as functions of $k \tau$. In addition, combining the relation $\Pi_{r}=0$ with Eq. (3.4), we obtain $\Phi=-\Psi$. In the following, we use this relation to eliminate $\Phi$.

In order to discuss the evolutions of the fluctuations, it is important to clarify the equation-of-state parameters of the individual components in the universe. If all the components behave as the relativistic or the non-relativistic matter, evolutions of the perturbations are described by Eqs. (3.7) - (3.10). In this case, it is convenient to distinguish the photon (or any other components) from the decay product of $\phi$ from that from the inflaton field, which we call $\gamma_{\phi}$ and $\gamma_{\chi}$, respectively. In order to consider $\delta_{\gamma_{\phi}}$ in the RD2

\footnotetext{
${ }^{\# 6}$ In fact, these photons are mixed each other and they cannot be defined separately. In other words, their velocity perturbations should be the same since they form a single fluid. Even so, the following arguments are unchanged as far as we consider the leading terms in the density perturbations since the velocity perturbation is at most $O(k \tau)$. In the following discussion, $\gamma_{\phi}$ and $\gamma_{\chi}$ should be understood as representatives of the components which are and are not generated from the decay product of $\phi$, respectively.
} 
epoch, we can neglect $\gamma_{\chi}$ since the CMB radiation at this epoch is dominantly from the $\phi$ field. Then, we find that, in the RD2 epoch, $\Psi$ and $\delta_{\gamma_{\phi}}$ become constant while $V_{\gamma_{\phi}}=O(k \tau)$ up to higher order corrections. Indeed, combining Eq. (3.3) with Eqs. (3.7) and (3.8), and using $\delta_{\text {tot }}=\delta_{\gamma_{\phi}}$ and $V_{\text {tot }}=V_{\gamma_{\phi}}$, we obtain $V_{\gamma_{\phi}}=-\frac{1}{2} k \tau \Psi_{\mathrm{RD} 2}^{(\delta \phi)}$ and

$$
\delta_{\gamma_{\phi}}=-2 \Psi_{\mathrm{RD} 2}^{(\delta \phi)}
$$

where $\Psi_{\mathrm{RD} 2}^{(\delta \phi)}$ is the metric perturbation induced by the primordial fluctuation of the amplitude of $\phi$. (In the following, the superscript " $(\delta \phi)$ " is for perturbations generated from the primordial fluctuation of $\phi$.) As we mentioned, $\Psi_{\mathrm{RD} 2}^{(\delta \phi)}$ is constant up to a correction of $O\left(k^{2} \tau^{2}\right)$.

Behavior of $\delta_{\gamma_{\chi}}$ is also easily understood. In discussing the effects of the primordial fluctuation of $\phi$, we neglect the initial fluctuation of the inflaton field and hence $\delta_{\gamma_{\chi}} \rightarrow 0$ in the deep RD1 epoch. In addition, from Eqs. (3.7) and (3.8), $V_{\gamma_{\chi}}$ becomes higher order in $k \tau$ than $\delta_{\gamma_{\chi}}$ and $\Psi$. Thus, we obtain

$$
\delta_{\gamma_{\chi}}=4 \Psi^{(\delta \phi)}
$$

The above relation holds in the RD1, $\phi \mathrm{D}$, and RD2 epochs up to corrections of $O\left(k^{2} \tau^{2}\right)$.

For the following discussion, it is convenient to define the entropy perturbation between $\gamma_{\phi}$ and $\gamma_{\chi}$ :

$$
S_{\phi \chi}^{(\delta \phi)} \equiv \frac{3}{4} \delta_{\gamma_{\phi}}-\frac{3}{4} \delta_{\gamma_{\chi}}=-\frac{9}{2} \Psi_{\mathrm{RD} 2}^{(\delta \phi)}
$$

For superhorizon modes, this entropy perturbation is a constant of time [23]. In addition, for example for the case where the $\phi$ field starts to oscillate during the $\chi \mathrm{D}$ epoch,

$$
S_{\phi \chi}^{(\delta \phi)}=\left[\frac{3}{4} \delta_{\gamma_{\phi}}-\frac{3}{4} \delta_{\gamma_{\chi}}\right]_{\mathrm{RD} 2}=\left[\delta_{\phi}-\frac{3}{4} \delta_{\gamma_{\chi}}\right]_{\phi \mathrm{D}, \mathrm{RD} 1}=\left[\delta_{\phi}-\delta_{\chi}\right]_{\chi \mathrm{D}},
$$

where the last equality holds for the epoch when $\phi$ and the inflaton field are both oscillating.

Density (and other) fluctuations in the RD2 epoch are generally parameterized by using $S_{\phi \chi}^{(\delta \phi)}$. If a component $X$ is generated from the decay product of $\phi$, then there is no entropy between the photon (i.e., $\gamma_{\phi}$ ) and $X$. On the contrary, if some other scalar field $\psi$ generates $X$, the entropy between the photon and $X$ is the same as $S_{\phi \chi}^{(\delta \phi)}$. Thus, if all the components in the universe are generated from $\phi$, the density fluctuations become purely adiabatic and

$$
\left[\delta_{\gamma}^{(\delta \phi)}\right]_{\mathrm{RD} 2}=\frac{4}{3}\left[\delta_{b}^{(\delta \phi)}\right]_{\mathrm{RD} 2}=\frac{4}{3}\left[\delta_{c}^{(\delta \phi)}\right]_{\mathrm{RD} 2}=-2 \Psi_{\mathrm{RD} 2}^{(\delta \phi)},
$$

where the subscripts $\gamma, b$, and $c$ are for the photon, baryon, and CDM, respectively. In this case, the isocurvature perturbation in the $\phi$ field is converted to the purely adiabatic 
density perturbation after the decay of $\phi[17$, 18, 19, 20]. On the contrary, if the baryon asymmetry is generated by the scalar field $\psi$, the entropy between the radiation and the baryon becomes $S_{\phi \chi}^{(\delta \phi)}$ and hence [20]

$$
\left[\delta_{\gamma}^{(\delta \phi)}\right]_{\mathrm{RD} 2}=\frac{4}{3}\left[\delta_{c}^{(\delta \phi)}\right]_{\mathrm{RD} 2}=-2 \Psi_{\mathrm{RD} 2}^{(\delta \phi)}, \quad\left[\delta_{b}^{(\delta \phi)}\right]_{\mathrm{RD} 2}=\frac{3}{4}\left[\delta_{\gamma}^{(\delta \phi)}\right]_{\mathrm{RD} 2}+\frac{9}{2} \Psi_{\mathrm{RD} 2}^{(\delta \phi)}
$$

and in the case where $\psi$ is responsible for the CDM while the baryon number is somehow generated from the decay product of $\phi$,

$$
\left[\delta_{\gamma}^{(\delta \phi)}\right]_{\mathrm{RD} 2}=\frac{4}{3}\left[\delta_{b}^{(\delta \phi)}\right]_{\mathrm{RD} 2}=-2 \Psi_{\mathrm{RD} 2}^{(\delta \phi)}, \quad\left[\delta_{c}^{(\delta \phi)}\right]_{\mathrm{RD} 2}=\frac{3}{4}\left[\delta_{\gamma}^{(\delta \phi)}\right]_{\mathrm{RD} 2}+\frac{9}{2} \Psi_{\mathrm{RD} 2}^{(\delta \phi)}
$$

In addition, if the baryon and the CDM are both generated from sources other than $\phi$, we obtain

$$
\left[\delta_{\gamma}^{(\delta \phi)}\right]_{\mathrm{RD} 2}=-2 \Psi_{\mathrm{RD} 2}^{(\delta \phi)}, \quad\left[\delta_{b}^{(\delta \phi)}\right]_{\mathrm{RD} 2}=\left[\delta_{c}^{(\delta \phi)}\right]_{\mathrm{RD} 2}=\frac{3}{4}\left[\delta_{\gamma}^{(\delta \phi)}\right]_{\mathrm{RD} 2}+\frac{9}{2} \Psi_{\mathrm{RD} 2}^{(\delta \phi)}
$$

It is important to notice that, for the cases given in Eqs. (3.17) - (3.19), the isocurvature perturbation is correlated with the adiabatic perturbation.

In the case with the $\psi$ field which is responsible for the baryon or the CDM, more careful considerations are needed. Adopting the parabolic potential for $\psi$, the fluctuation of the $\psi$ field obeys

$$
\ddot{\delta} \psi+3 H \dot{\delta} \dot{\psi}+\left[k^{2}\left(\frac{a}{a_{0}}\right)^{2}+m_{\psi}^{2}\right] \delta \psi=-2 m_{\psi}^{2} \psi \Psi+\dot{\psi}(\dot{\Psi}-3 \dot{\Phi}) .
$$

Solving this equation, we see that the fluctuation of $\psi$ at the time of $H \sim m_{\psi}$ is of the order of $[\psi \Psi]_{H \sim m_{\psi}}$. If the $\psi$ field starts to oscillate in the RD1 epoch, energy density of $\psi$ behaves as that of non-relativistic components even when the metric perturbation $\Psi$ is negligibly small. In this case, $[\psi \Psi]_{H \sim m_{\psi}}$ is small enough to be neglected and the former discussion applies. If $\psi$ starts to oscillate in the $\phi \mathrm{D}$ epoch, on the contrary, the situation changes. In this case, the $\psi$ field is slowly rolling when the $\phi \mathrm{D}$ epoch starts and $\delta \psi$ at the time of the $\phi$-domination is negligibly small. Then, solving Eq. (3.20) for the superhorizon mode, we obtain

$$
\delta \psi_{\phi \mathrm{D}}^{(\delta \phi)}=\dot{\psi} \Psi_{\phi \mathrm{D}}^{(\delta \phi)} t
$$

where we used the fact that $\dot{\Psi}$ and $\dot{\Phi}$ are small enough to be neglected in the $\phi \mathrm{D}$ epoch. With Eq. (3.21), we can calculate the density fluctuation of $\psi$ as $\left[\rho_{\psi} \delta_{\psi}\right]_{\phi \mathrm{D}}=-3 H \dot{\psi}^{2} \Psi_{\phi \mathrm{D}}^{(\delta \phi)} t$. Importantly, the relation (3.21) holds even after the $\psi$ field starts to oscillate, as far as $H$ is substantially larger than the decay rate of $\psi$. So, we can calculate the entropy between $\psi$ and $\phi$ in the $\phi \mathrm{D}$ epoch after the $\psi$ field starts to oscillate; using the relation $[\delta \phi]_{\phi \mathrm{D}}=-2 \Psi_{\phi \mathrm{D}}^{(\delta \phi)}$ and $\left\langle\dot{\psi}^{2}\right\rangle=\rho_{\psi}$, we see that the entropy between $\psi$ and $\phi$ vanishes. Thus, if the $\psi$ field is slow-rolling when the $\phi \mathrm{D}$ epoch starts, density perturbation of 
the component generated from $\psi$ becomes adiabatic. If the $\psi$ field starts to oscillate just at the time when the $\phi \mathrm{D}$ epoch starts, the correlated mixture of the adiabatic and isocurvature perturbations is generated but the ratio of the metric perturbation to the entropy perturbation may deviate from that given in Eqs. (3.17) - (3.19).

Before closing this section, let us estimate the size of $S_{\phi \chi}^{(\delta \phi)}$. As we mentioned before, primordial fluctuation of the $\phi$ field is probably generated during the inflation. In this case, calculating the two-point function of $\phi$ in the de Sitter background, we obtain

$$
\langle 0|\delta \phi(t, \vec{x}) \delta \phi(t, \vec{y})| 0\rangle_{\mathrm{inf}}=\int \frac{d k}{k}|\delta \phi(t, \vec{k})|^{2} e^{i \vec{k}(\vec{x}-\vec{y})},
$$

where the Fourier amplitude for the case of $m_{\phi} \ll H_{\text {inf }}$ is given by

$$
\delta \phi(t, \vec{k})=\left(\frac{k}{2 a H_{\mathrm{inf}}}\right)^{2 m_{\phi}^{2} / 3 H_{\mathrm{inf}}^{2}}\left[\frac{H_{\mathrm{inf}}}{2 \pi}\right]_{k=a H_{\mathrm{inf}}} .
$$

(The subscript "inf" means that the correlation function is evaluated during the inflation.) Once the wavelength becomes longer than the horizon scale, the scalar-field fluctuation obeys the following equation:

$$
\ddot{\delta \phi}+3 H \dot{H} \phi+\left[k^{2}\left(\frac{a}{a_{0}}\right)^{2}+m_{\phi}^{2}\right] \delta \phi=-2 m_{\phi}^{2} \phi \Psi+\dot{\phi}(\dot{\Psi}-3 \dot{\Phi}) .
$$

First, let us consider the case where $\phi_{\text {init }} \ll M_{*}$. In this case, when the $\phi$ field starts to move, the energy density of $\phi$ is much smaller than the total energy density of the universe. In studying the density perturbations generated by the primordial fluctuations of $\phi$, the metric perturbations are negligibly small until the energy density of $\phi$ becomes comparable to the total energy density of the universe. In this case, we can neglect the terms containing the metric perturbations in Eq. (3.24). Furthermore, the $k^{2}$-term is also irrelevant for the superhorizon perturbations. Then, we can see that, with the above approximations, $\delta \phi$ obeys the same equation as the zero-mode. Neglecting the decaying mode, we obtain the relation

$$
\delta \phi(t, k)=\frac{\delta \phi_{\text {init }}(k)}{\phi_{\text {init }}} \phi(t)
$$

where $\delta \phi_{\text {init }}$ is the initial value of the fluctuation of $\phi$, which is $H_{\text {inf }} / 2 \pi$. In addition, $\delta_{\chi} \rightarrow 0$ (and hence $\delta_{\gamma_{\chi}} \rightarrow 0$ ) in the early universe, and hence the initial value of $S_{\phi \chi}^{(\delta \phi)}$ is estimated as

$$
S_{\phi \chi}^{(\delta \phi)}(k)=\frac{\delta \rho_{\phi}(t, k)}{\rho_{\phi}(t)}-\delta_{\chi}(t)=\frac{2 \delta \phi_{\text {init }}(k)}{\phi_{\text {init }}}
$$

Thus, the entropy $S_{\phi \chi}^{(\delta \phi)}$ increases as the initial amplitude of $\phi$ decreases. If the initial amplitude of $\phi$ becomes close to $M_{*}$, the energy density of $\phi$ becomes comparable to the 
total energy density of the universe. Then, the metric perturbation $\Psi$ becomes comparable to the density perturbation of the $\phi$ field $\delta_{\phi}$. In this case, sizable $\Psi$ generates the density perturbations of other components and hence the relation (3.26) may not hold. Even in this case, however, Eq. (3.26) provides a good order-of-estimation of the primordial entropy perturbation between $\phi$ and $\chi$ at the time when the $\phi$ field starts to oscillate; as far as there is no inflation caused by $\phi, S_{\phi \chi}^{(\delta \phi)} \sim O\left(\delta \phi_{\text {init }} / M_{*}\right)$ when $\phi_{\text {init }} \sim M_{*}$.

\section{CMB Angular Power Spectrum: Overview}

In this section, we study the CMB anisotropy generated from fluctuations of various scalar fields. The CMB anisotropy is characterized by the angular power spectrum $C_{l}$ which is defined as

$$
\left\langle\Delta T(\vec{x}, \vec{\gamma}) \Delta T\left(\vec{x}, \vec{\gamma}^{\prime}\right)\right\rangle_{\vec{x}}=\frac{1}{4 \pi} \sum_{l}(2 l+1) C_{l} P_{l}\left(\vec{\gamma} \cdot \vec{\gamma}^{\prime}\right)
$$

with $\Delta T(\vec{x}, \vec{\gamma})$ being the temperature fluctuation of the CMB radiation pointing to the direction $\vec{\gamma}$ at the position $\vec{x}$ and $P_{l}$ is the Legendre polynomial. The angular power spectrum $C_{l}$ depends on the properties of the primordial density fluctuations and hence it provides probes for studying how the density perturbations are generated in the early universe.

In the scenario we consider, there are three possible sources of the density perturbations; the primordial fluctuations of the scalar fields $\chi, \phi$, and $\psi$. Since there is no correlation among these fields, the CMB anisotropies from these fluctuations are uncorrelated and the resultant $\mathrm{CMB}$ power spectrum is given in the form

$$
C_{l}=C_{l}^{(\delta \chi)}+C_{l}^{(\delta \phi)}+C_{l}^{(\delta \psi)}
$$

where $C_{l}^{(\delta \chi)}, C_{l}^{(\delta \phi)}$ and $C_{l}^{(\delta \psi)}$ are contributions from the primordial fluctuations of the inflaton field $\chi$, the $\phi$ field and the $\psi$ field, respectively.\#7

The inflaton contribution $C_{l}^{(\delta \chi)}$ is the same as the adiabatic result while $C_{l}^{(\delta \psi)}$ becomes the purely isocurvature one. Thus, in the following, we study the properties of $C_{l}^{(\delta \phi)}$.

Effects of the primordial fluctuation of the $\phi$ field depend on the scenario as we discussed. If all the components in the universe originate to $\phi$, then the density perturbations become adiabatic. In this case, it is difficult to experimentally distinguish $C_{l}^{(\delta \phi)}$ from the adiabatic CMB power spectrum generated from the inflaton fluctuations. Even in this case, however, there are important implications in studying scenarios of generating the cosmic density fluctuations, like inflation, as will be discussed in Section 5 .

On the contrary, if the scalar field $\psi$ exists which is responsible for the baryon or the CDM, $C_{l}^{(\delta \phi)}$ may become different from the angular power spectrum from the purely

\footnotetext{
${ }^{\# 7}$ If all the components in the universe are generated from the decay product of $\phi$, the $\psi$ field is irrelevant and $C_{l}^{(\delta \psi)}$ does not exist.
} 
adiabatic density perturbation since the adiabatic and isocurvature perturbations can be generated with cross-correlation. Although we have considered the entropy perturbations in the baryonic and the CDM sectors separately in Eqs. (3.17) - (3.19), we found that the resultant $C_{l}^{(\delta \phi)}$ is primarily determined by the entropy between the non-relativistic matter and radiation; if we parameterize the density perturbation of the non-relativistic component as

$$
\left[\delta_{m}^{(\delta \phi)}\right]_{\mathrm{RD} 2} \equiv\left[\left(\Omega_{b} / \Omega_{m}\right) \delta_{b}^{(\delta \phi)}+\left(\Omega_{c} / \Omega_{m}\right) \delta_{c}^{(\delta \phi)}\right]_{\mathrm{RD} 2}=\frac{3}{4}\left[\delta_{\gamma}^{(\delta \phi)}\right]_{\mathrm{RD} 2}+\kappa_{m} \Psi_{\mathrm{RD} 2}^{(\delta \phi)},
$$

where $\Omega_{b}, \Omega_{c}$ and $\Omega_{m}$ are the (present) density parameters for the baryon, the CDM, and the non-relativistic component (and hence $\Omega_{m}=\Omega_{b}+\Omega_{c}$ ), the parameter $\kappa_{m}$ determines the shape of $C_{l}^{(\delta \phi)}$. With the relations (3.17), (3.18), and (3.19), $\kappa_{m}$ becomes $\frac{9}{2}\left(\Omega_{b} / \Omega_{m}\right)$, $\frac{9}{2}\left(\Omega_{c} / \Omega_{m}\right)$, and $\frac{9}{2}$, respectively. On the contrary, if the $\psi$ field is slow-rolling when the $\phi \mathrm{D}$ epoch starts, $\kappa_{m}$ vanishes. In addition, if $\psi$ accidentally starts to oscillate just at the beginning of the $\phi \mathrm{D}$ epoch, $\kappa_{m}$ may take any value between 0 and $\frac{9}{2}$. This may happen, however, when the expansion rate at the beginning of the $\phi \mathrm{D}$ epoch accidentally becomes comparable to $m_{\psi}$.

In Fig. 3, we plot the angular power spectrum with the correlated mixture of the adiabatic and isocurvature perturbations in the baryonic and/or CDM sector, i.e., the cases with the relations given in Eqs. (3.17) - (3.19). Here, we consider the flat universe with $\Omega_{b} h^{2}=0.019, \Omega_{m}=0.3$, and $h=0.65$ [24], where $h$ is the Hubble constant in units of $100 \mathrm{~km} / \mathrm{sec} / \mathrm{Mpc}$. For comparison, we also plot the angular power spectrum for the purely adiabatic and isocurvature cases (i.e., $C_{l}^{(\delta \chi)}$ and $C_{l}^{(\delta \psi)}$ ). As one can see, the CMB angular power spectrum strongly depends on properties of the primordial density perturbations. If there exists correlated entropy between the baryon and other components with the relation (3.17), negative interference between the adiabatic and isocurvature; perturbations suppresses $C_{l}$ at lower multipole while the effect of the isocurvature perturbation becomes too small to affect the structure at high multipole. As a result, the angular power spectrum is enhanced at the high multipole rather than at the low multipole. If the effect of the entropy perturbation becomes more efficient, then $C_{l}^{(\delta \psi)}$ at high multipole is suppressed relative to that at low multipole like in the purely isocurvature case. This happens when the entropy perturbation is in the CDM component with the condition given in Eq. (3.18). In addition, with the relation given in Eq. (3.19), the correlated entropy becomes more effective than the case where only the CDM sector has the correlated entropy. Then, the acoustic peaks are more suppressed relative to the Sachs-Wolfe (SW) tail.

As discussed in the previous section, in the general case, the $\kappa_{m}$ parameter may vary between 0 and $\frac{9}{2}$, and hence the CMB anisotropy generated from the primordial fluctuation of $\phi$ changes its behavior as $\kappa_{m}$ varies. In particular, if $\delta \phi_{\text {init }}$ is the only source of the cosmic density perturbations, too large $\kappa_{m}$ results in angular power spectrum which is inconsistent with observations. In this case, we can derive a constraint on the $\kappa_{m}$ parameter.

In order to derive the constraint, we calculate the goodness-of-fit parameter $\chi^{2}=$ $-2 \ln L$, where $L$ is the likelihood function, as a function of $\kappa_{m}$. In our calculation, the 


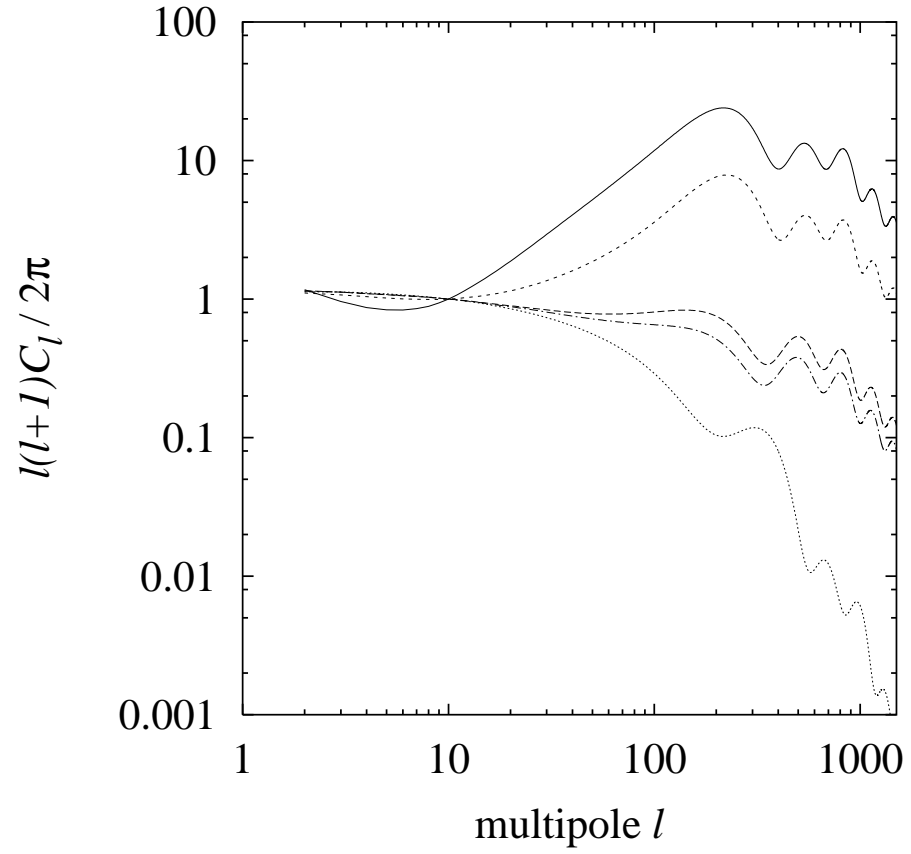

Figure 3: The angular power spectrum with correlated mixture of the adiabatic and isocurvature perturbations in the baryonic sector (solid line), in the CDM sector (longdashed line), and in the baryonic and CDM sectors (dot-dashed line). (See Eqs. (3.17), (3.18), and (3.19), respectively.) We also show the CMB angular power spectrum in the purely adiabatic (short-dashed line) and isocurvature density perturbations (dotted line), i.e., $C_{l}^{(\delta \chi)}$ and $C_{l}^{(\delta \psi)}$. We consider the flat universe with $\Omega_{b} h^{2}=0.019, \Omega_{m}=0.3$, and $h=0.65$, and the initial power spectral indices for primordial density perturbations are all assumed to be 1 (i.e., we adopt scale-invariant initial power spectra). The overall normalizations are taken as $\left[l(l+1) C_{l} / 2 \pi\right]_{l=10}=1$.

offset log-normal approximation is used [25]. We use a data set consisting of 65 data points; 24 from COBE/DMR [1] in the range $2 \leq l \leq 25,19$ from BOOMERanG [2] in the range $76 \leq l \leq 1025,13$ from MAXIMA [3] in the range $36 \leq l \leq 1235$, and 9 from DASI [4] in the range $104 \leq l \leq 864$. From the figure, we can read off the constraint on $\kappa_{m}$. Requiring $\chi \leq 84$, which corresponds to $95 \%$ C.L. allowed region for the $\chi^{2}$ statistics with 64 degrees of freedom, we obtain the constraint $\kappa_{m} \leq 0.3$ for $\Omega_{m}=0.3$, and $1.7 \leq \kappa_{m} \leq 2.0$ for $\Omega_{m}=0.2$. Interestingly, the $\chi^{2}$ variable is relatively suppressed when $\kappa_{m} \sim 2$. This is because the heights of the acoustic peaks relative to the SW tail once increase then decrease as we increase the $\kappa_{m}$ parameter. In particular, when $\kappa_{m} \sim 2$, the heights of the acoustic peaks relative to the SW tail become similar to those from the adiabatic density perturbations which well agrees with the observations. Of course, if the effects of $C_{l}^{(\delta \chi)}$ or $C_{l}^{(\delta \psi)}$ become important, the above constraint on $\kappa_{m}$ does not apply. 


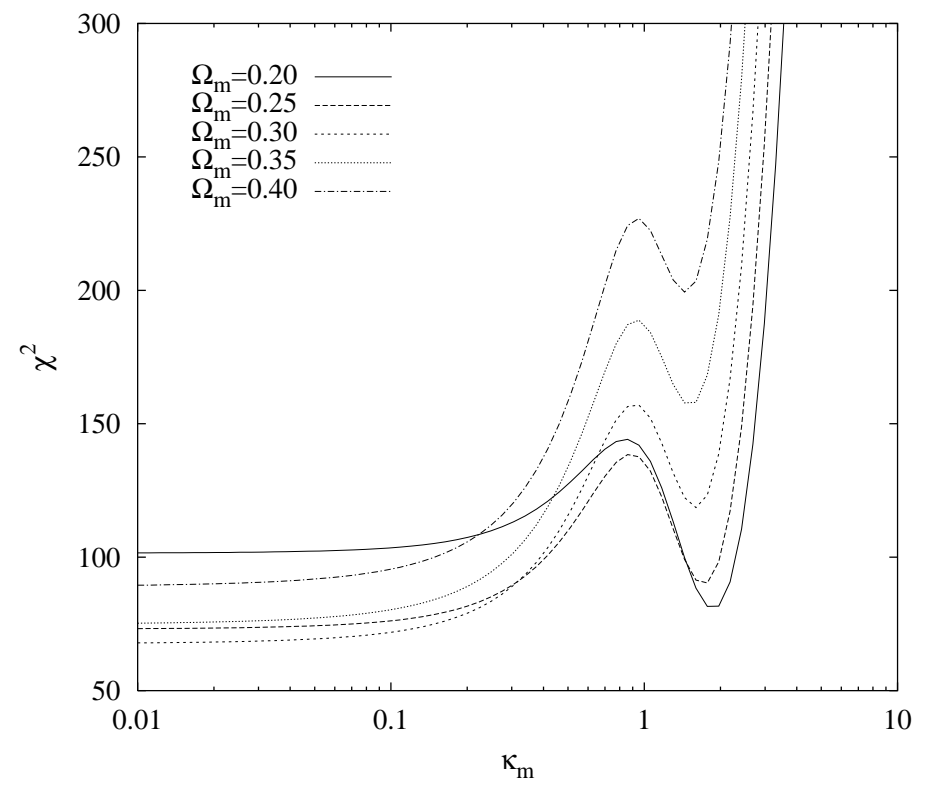

Figure 4: $\chi^{2}$ as a function of $\kappa_{m}$. We take $\Omega_{b} h^{2}=0.019, h=0.65$, and the values of $\Omega_{m}$ are shown in the figure. The flat universe is assumed. Notice that the $\kappa_{m}$ parameter is smaller than $\frac{9}{2}$ in our scenario.

\section{Case without Entropy Perturbations}

If all the component of the universe (i.e., the photon, baryon, CDM, neutrino, and so on) are generated from the decay product of $\phi$, there is no entropy between any of two components. In this case, the resultant angular power spectrum is given in the form

$$
C_{l}=C_{l}^{(\delta \chi)}+C_{l}^{(\delta \phi)}
$$

Importantly, the two contributions $C_{l}^{(\delta \chi)}$ and $C_{l}^{(\delta \phi)}$ are both from adiabatic perturbations. Thus, for the case where scale dependence of the sources of $C_{l}^{(\delta \chi)}$ and $C_{l}^{(\delta \phi)}$, i.e., $\Psi^{(\delta \chi)}$ and $S_{\phi \chi}^{(\delta \phi)}$, are the same, $C_{l}^{(\delta \phi)} \propto C_{l}^{(\delta \chi)}$. In this case, it is impossible to test the scenario with $\phi$ using experimental data.

In general, however, the scale dependences of $\Psi^{(\delta \chi)}$ and $S_{\phi \chi}^{(\delta \phi)}$ are different. In the slowroll inflation scenario, $\Psi^{(\delta \chi)}$ is generated from the fluctuation of the inflaton amplitude and is given by [26]

$$
\Psi_{\mathrm{RD} 2}^{(\delta \chi)}=\frac{4}{9}\left[\frac{H_{\mathrm{inf}}}{2 \pi} \frac{3 H_{\mathrm{inf}}^{2}}{V_{\mathrm{inf}}^{\prime}}\right]_{k=a H_{\mathrm{inf}}}
$$

where $V_{\text {inf }}^{\prime} \equiv \partial V_{\text {inf }} / \partial \chi$ with $V_{\text {inf }}$ being the inflaton potential. On the contrary, $S_{\phi \chi}^{(\delta \phi)}$ is 
related to the fluctuation of the amplitude of $\phi$, as seen in Eq. (3.26), and hence

$$
S_{\phi \chi}^{(\delta \phi)}=\frac{2}{\phi_{\text {init }}}\left[\frac{H_{\mathrm{inf}}}{2 \pi}\right]_{k=a H_{\mathrm{inf}}} .
$$

In many models of slow-roll inflation, the expansion rate $H_{\text {inf }}$ is almost constant during the inflation. On the contrary, the slope of the inflation potential $V_{\text {inf }}^{\prime}$ may significantly vary. As a result, $S_{\phi \chi}^{(\delta \phi)}$ becomes (almost) scale independent while $\Psi^{(\delta \chi)}$ may have sizable scale dependence. Since the currently measured CMB power spectrum suggests (almost) scale invariant primordial density perturbation in the conventional scenario, inflation models are excluded if $\Psi^{(\delta \chi)}$ has too strong scale dependence [27].

If the $\phi$ field exists, however, the situation may change. Since the variable $S_{\phi \chi}^{(\delta \phi)}$ is expected to be (almost) scale invariant, we can relax the constraint on the inflation models if $C_{l}^{(\delta \phi)}$ becomes significantly large, which happens when $S_{\phi \chi}^{(\delta \phi)} \gtrsim \Psi^{(\delta \chi)}$. As shown in Eq. (3.26), $S_{\phi \chi}^{(\delta \phi)}$ is inversely proportional to $\phi_{\text {init. }}$. Thus, if the initial amplitude of $\phi$ is suppressed, this may happen. In particular, if $C_{l}^{(\delta \phi)} \gg C_{l}^{(\delta \chi)}$, the present cosmic density fluctuations are totally from the primordial fluctuation of $\phi$ [18, 19, 20]. (Such a scenario is sometimes called "curvaton scenario."

In addition, this mechanism of converting the isocurvature density perturbation in the scalar field to the adiabatic density perturbation may have some significance in the pre-bigbang [15] and ekpyrotic [16] scenario. In Refs. [18, 19], it was discussed that the present cosmological density perturbations may be generated from the primordial fluctuation of some axion-like fields in the pre-big-bang and ekpyrotic scenarios.

\section{Cases with Entropy Perturbations}

In this section, we discuss the CMB angular power spectrum in cases with entropy perturbations. In particular, we study the effects of the correlated entropy perturbation generated in the cases where the baryonic or the CDM component is generated from $\psi$, not from $\phi$. As we mentioned, if the $\psi$ field starts to oscillate before the $\phi \mathrm{D}$ epoch starts, the relation (3.17), (3.18) or (3.19) holds. On the contrary, if $\psi$ is slow-rolling at the time of the $\phi$-domination, density perturbations become adiabatic. Thus, in this section, we consider the most typical relations (3.17) - (3.19) when the adiabatic and isocurvature perturbations exist with cross-correlation. In the general case, as we mentioned, the relation between the adiabatic and isocurvature perturbations may deviate from Eqs. (3.17) - (3.19). This may happen, however, only if the expansion rate at the beginning of the $\phi \mathrm{D}$ epoch becomes accidentally close to $m_{\psi}$, and we will not consider such a case in this paper. (See [29] for such a case.)

As discussed in the previous section, the CMB power spectrum is given in the form

$$
C_{l}=C_{l}^{(\delta \chi)}+C_{l}^{(\delta \phi)}+C_{l}^{(\delta \psi)} .
$$

\footnotetext{
${ }^{\# 8}$ For recent discussions on the curvaton scenario, see [28].
} 
Here, $C_{l}^{(\delta \chi)}$ is from the inflaton fluctuation which is usually parameterized by the metric perturbation generated by the inflaton fluctuation (in the RD2 epoch), $\Psi_{\mathrm{RD} 2}^{(\delta \chi}$. In addition, $C_{l}^{(\delta \phi)}$ and $C_{l}^{(\delta \psi)}$ are parameterized by the entropy perturbations in the baryon or the CDM. To parameterize these contributions, we define the ratios

$$
R_{b} \equiv S_{b \gamma}^{(\delta \phi)} / \Psi_{\mathrm{RD} 2}^{(\delta \chi)}, \quad R_{c} \equiv S_{c \gamma}^{(\delta \phi)} / \Psi_{\mathrm{RD} 2}^{(\delta \chi},
$$

and

$$
\alpha_{b} \equiv S_{b \gamma}^{(\delta \psi)} / \Psi_{\mathrm{RD} 2}^{(\delta \chi)}, \quad \alpha_{c} \equiv S_{c \gamma}^{(\delta \psi)} / \Psi_{\mathrm{RD} 2}^{(\delta \chi},
$$

where $S_{b \gamma}^{(\delta \phi)}\left(S_{c \gamma}^{(\delta \phi)}\right)$ is the entropy between the baryon and the photon (between the CDM and the photon) generated from the primordial fluctuation of $\phi$, and $S_{b \gamma}^{(\delta \psi)}$ and $S_{c \gamma}^{(\delta \psi)}$ are those from $\delta \psi$. (We adopt Eqs. (3.17) and (3.18), and hence $S_{b \gamma}^{(\delta \psi)}$ and $S_{c \gamma}^{(\delta \psi)}$ are equal to $\frac{9}{2} \Psi_{\mathrm{RD} 2}^{(\delta \phi)}$ if they are non-vanishing.) The shape of the CMB angular power spectrum depends on the values of these parameters.

The expressions for $\Psi_{\mathrm{RD} 2}^{(\delta \chi)}$ and $S_{\phi \chi}^{(\delta \phi)}$ are given in Eqs. (5.2) and (5.3), respectively, and hence, if $\psi$ starts to oscillate before the $\phi \mathrm{D}$ epoch starts, the $R$-parameters are given as

$$
R_{b, c}=\frac{3}{2}\left[\frac{V_{\mathrm{inf}}^{\prime}}{\phi_{\mathrm{init}} H_{\mathrm{inf}}^{2}}\right]_{k=a H_{\mathrm{inf}}} .
$$

Hence $R_{b, c}$ is model- and scenario-dependent; it depends on the scale of inflation, shape of the inflaton potential, and initial amplitude of $\phi$. For example in the chaotic inflation model with the parabolic potential $V_{\mathrm{inf}}=\frac{1}{2} m_{\chi}^{2} \chi^{2}$, the above expression becomes

$$
\left[R_{b, c}\right]_{\text {chaotic }}=\left[\frac{9 M_{*}^{2}}{\phi_{\text {init }} \chi}\right]_{k=a H_{\text {inf }}} .
$$

Using the fact that the inflaton amplitude at the time of the horizon crossing of the COBE scale is $\chi \simeq 15 M_{*}$ in the chaotic inflation model, $\left[R_{b, c}\right]_{\text {chaotic }} \simeq 0.6 M_{*} / \phi_{\text {init }}$. Of course, the values of $R_{b}$ and $R_{c}$ depend on the model of inflation, and they vary if we consider different class of inflation models.

The $\alpha$-parameters are also estimated in the similar way, and the result is given from the expression for the $R$-parameters by replacing $\phi \rightarrow \psi$.

\subsection{Uncorrelated Entropy Perturbation}

Although our main subject is to investigate effects of the fluctuation of the $\phi$ field, it is instructive to study effects of the uncorrelated isocurvature perturbations. Thus, let us first consider the case with uncorrelated isocurvature perturbations (i.e., case with $\left.R_{b}=R_{c}=0\right)$. 




Figure 5: The CMB angular power spectrum with the mixed baryonic isocurvature and adiabatic density perturbations with $\alpha_{b}=0$ (solid line), 3 (long-dashed line) and 5 (shortdashed line). The cosmological parameters are the same as those in Fig. 3. The overall normalizations are arbitrary.

In this case, the resultant CMB power spectrum is given in the form

$$
C_{l}=C_{l}^{(\delta \chi)}+\alpha^{2}\left[C_{l}^{(\delta \psi)}\right]_{\alpha=1}
$$

where $\alpha$ here is $\alpha_{b}$ or $\alpha_{c}$ depending on the scenario.

In Fig. 5, we plot the total angular power spectrum for several values of $\alpha_{b}$ (with $\left.\alpha_{c}=0\right)$. As can be seen, as the effects of the isocurvature mode become more significant, the power spectrum at the high multipole is more suppressed relative to $C_{l}$ at small $l$. In addition, since the effects of $C_{l}^{(\delta \psi)}$ at high multipole are very small, the structure of the acoustic peaks are almost unchanged from the adiabatic result unless the $\alpha_{b}$-parameter becomes extremely large.

Since the isocurvature mode changes the shape of the CMB power spectrum, upper bounds on the $\alpha$-parameters can be obtained; if the $\alpha$-parameters are too large, the acoustic peaks are so suppressed that the CMB power spectrum becomes inconsistent with the observations. $\$$ PI

As in the previous case, we calculate the $\chi^{2}$ variable as a function of $\alpha_{b}$, and the results are shown in Fig. 6. From the figure, we see that too large $\alpha_{b}$ is disfavored by the current observations; taking $\Omega_{m}=0.3$, which gives the most conservative upper bound on $\alpha_{b}$, the constraint $\alpha_{b} \leq 4.5$ at $95 \%$ C.L. is obtained.

\footnotetext{
${ }^{\# 9}$ For other discussions on the constraints on the uncorrelated isocurvature perturbations, see [30].
} 


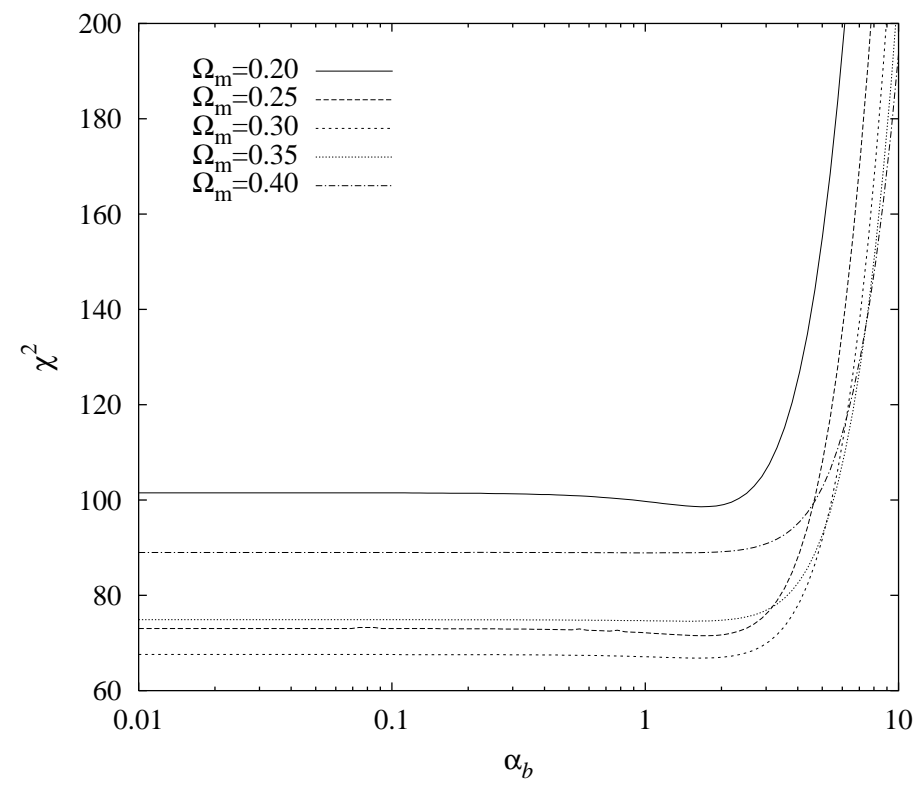

Figure 6: $\chi^{2}$ as a function of $\alpha_{b}$. Here we take $\Omega_{b} h^{2}=0.019, h=0.65$, and the values of $\Omega_{m}$ are shown in the figure. The flat universe is assumed.

The CMB angular power spectrum with $\alpha_{c} \neq 0$ can be also studied. In fact, the above results with $\alpha_{b} \neq 0$ can be translated to the CDM isocurvature cases; the CMB angular power spectrum for the baryonic and the CDM isocurvature cases are the same if $\Omega_{b} \alpha_{b}=\Omega_{c} \alpha_{c}$; using this relation, we can also derive the upper bound on $\alpha_{c}$. Thus, in any case, the heights of the acoustic peaks decrease relative to the SW tail as the (uncorrelated) isocurvature fluctuation contaminates.

\subsection{Correlated Entropy Perturbation in the Baryonic Sector}

Now, we study effects of the correlated entropy fluctuation in the baryonic sector (i.e., case with $R_{b} \neq 0$ ). Here, we assume other entropy perturbations to vanish (i.e., $R_{c}=$ $\alpha_{b}=\alpha_{c}=0$ ). The general case will be discussed later.FIf In this case, the total angular power spectrum is given in the form

$$
C_{l}=C_{l}^{(\delta \chi)}+R_{b}^{2}\left[C_{l}^{(\delta \phi)}\right]_{R_{b}=1} .
$$

Thus, we expect that, as the $R_{b}$-parameter increases, the angular power spectrum at the high multipole is more enhanced relative to $C_{l}$ at low multipole.

\footnotetext{
${ }^{\# 10}$ Notice that the $\alpha$-parameters vanish, for example, for the case where the effective mass of the $\psi$ field becomes comparable to (or larger than) the expansion rate during the inflation.
} 


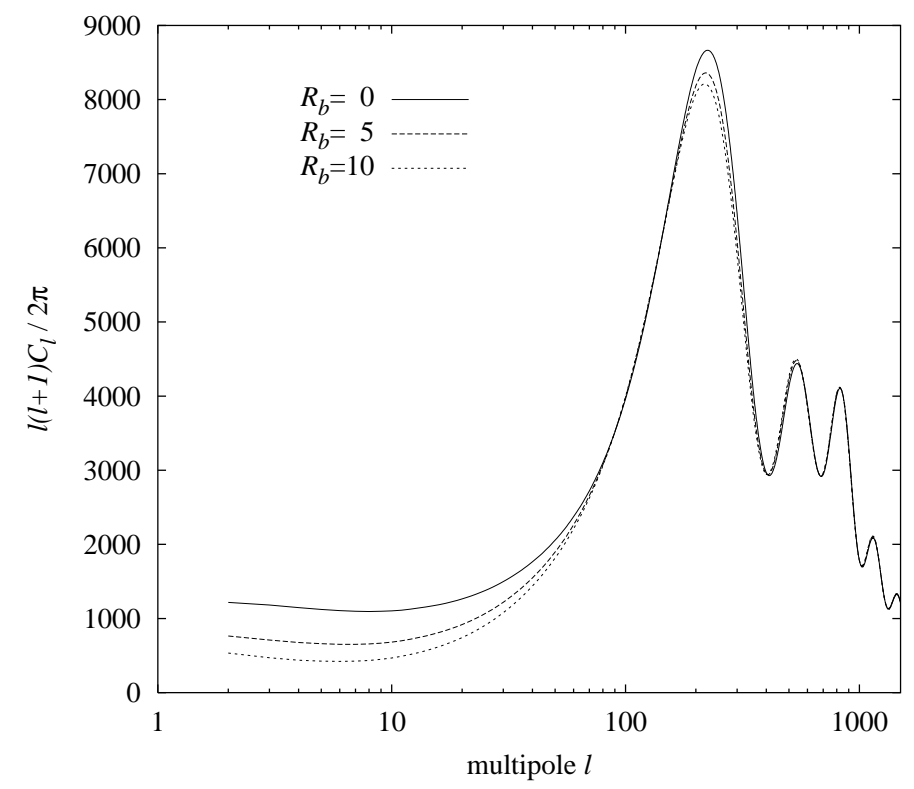

Figure 7: The CMB angular power spectrum for the case of $\alpha_{b}=0$. Here we take $R_{b}=0$ (solid line), 5 (long-dashed line) and 10 (short-dashed line). The cosmological parameters are the same as those in Fig. 3. The overall normalizations are arbitrary.

In Fig. 7, we plot the resultant angular power spectrum with several values of $R_{b}$. As expected, $C_{l}$ at the high multipole is more enhanced relative to that at low ones as the $R_{b^{-}}$ parameter increases, and hence it can be a signal of the late-decaying scalar condensation.

In fact, if the $R_{b}$-parameter is too large, the angular power spectrum at high multipole is too enhanced, which becomes inconsistent with the currently available data. For the quantitative discussion, we calculate the goodness-of-fit parameter as the previous case as a function of $R_{b}$. The results are shown in Fig. 8 for several sets of the cosmological parameters. The figure shows that too large $R_{b}$ becomes inconsistent with the observations if the total angular power spectrum is given by the sum of $C_{l}^{(\delta \chi)}$ and $C_{l}^{(\delta \phi)}$. Numerically, even adopting the most conservative constraint, $R_{b} \geq 4.5$ is excluded at $95 \%$ C.L.

Before closing this subsection, we would like to comment on the case where the cosmic density perturbations are totally from the primordial fluctuation of $\phi$, i.e., the case of the curvaton scenario. In this class of scenario, if the curvaton field $\phi$ does not generate the baryon (or the CDM), the adiabatic and isocurvature perturbations may be generated with cross-correlation with the relation given in Eq. (3.17) (or Eq. (3.18). . TH The curvaton scenario requires $C_{l}^{(\delta \phi)} \gg C_{l}^{(\delta \chi)}$ and hence $R_{b}$ (or $R_{c}$ ) becomes much larger than 1, which is already excluded by the current observations. $\left(R_{c} \gg 1\right.$ is also excluded as will be shown

\footnotetext{
${ }^{\# 11}$ In more complicated cases, this may not be the case. If some fraction of the baryon is from the decay product of $\phi$ while the rest is from $\psi$, for example, the relation given in Eq. (3.17) does not hold. In this paper, however, we do not consider such a complicated scenario.
} 


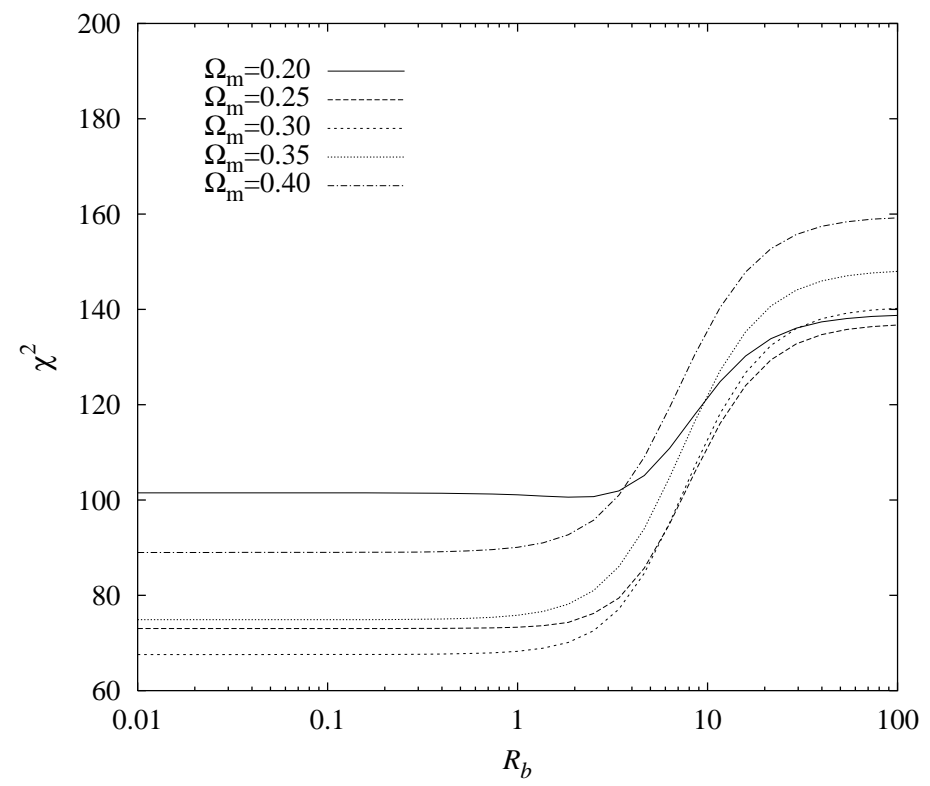

Figure 8: $\chi^{2}$ as a function of $R_{b}$. We take $\Omega_{b} h^{2}=0.019$ and $h=0.65$. The values of $\Omega_{m}$ are shown in the figures. The flat universe is assumed.

in the next subsection.) One caveat to this argument is that, if the $\psi$ field is so light that it starts to oscillate after the curvaton field dominates the universe, $R_{b}$ (or $R_{c}$ ) vanishes and such a scenario is consistent with the current data.

\subsection{Correlated Entropy Perturbation in the CDM Sector}

In this subsection, we consider the case with the correlated entropy perturbation in the CDM sector. Effects of the correlated entropy perturbation in the CDM sector is different from those in the baryonic sector. Since the density parameter for the CDM $\Omega_{c}$ is significantly larger than $\Omega_{b}$, entropy between the non-relativistic matter and the radiation becomes larger compared to the baryonic case. Then, the effects of the correlation between the adiabatic and isocurvature perturbations become less significant while the effect of the isocurvature perturbation itself becomes more important. As a result, as discussed in the previous section, if there exists the correlated entropy perturbation in the CDM sector with the relation (3.18), the acoustic peaks become lower relative to the SW tail. In Fig. 9, we show the resultant CMB angular power spectrum for several values of $R_{c}$ (with $\left.R_{b}=\alpha_{b}=\alpha_{c}=0\right)$. As $R_{c}$ increases, the acoustic peaks are more suppressed relative to the $C_{l}$ at lower multipole.

As in the previous cases, if the effect of the isocurvature perturbation becomes too effective, the resultant power spectrum becomes inconsistent with the observations. Thus, using the current experimental data, we can put an upper bound on the $R_{c}$-parameter. 


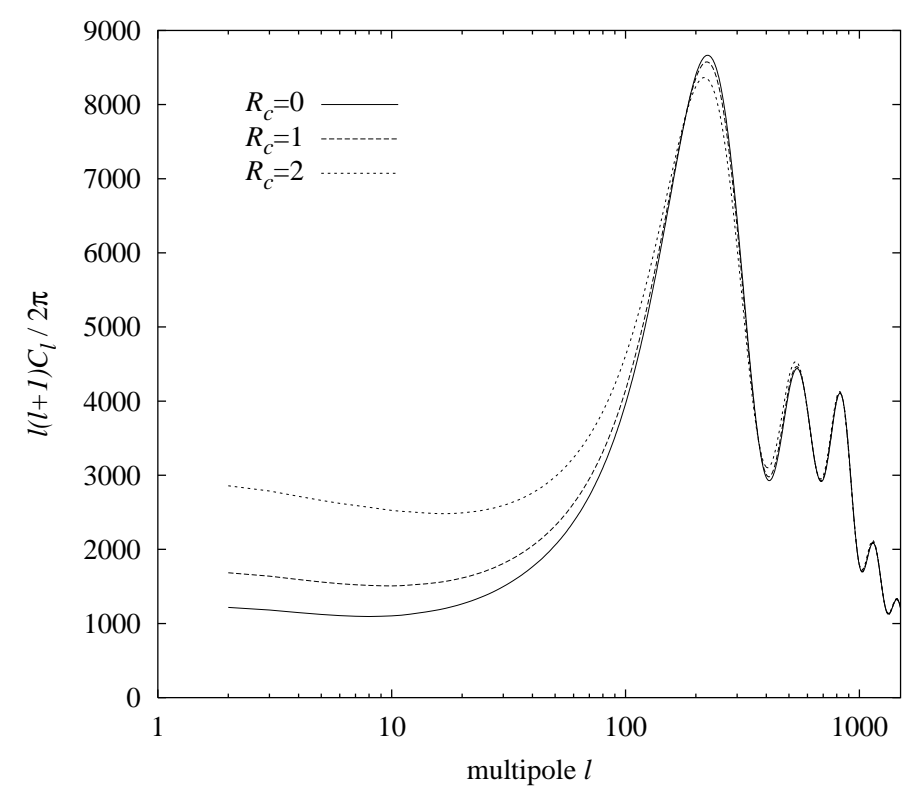

Figure 9: The CMB angular power spectrum for the case with correlated entropy perturbation in the CDM sector with $R_{c}=0$ (solid line), 1 (long-dashed line), and 2 (short-dashed line). The cosmological parameters are the same as Fig. 3. The overall normalization are arbitrary.

In Fig. 10, we plot the goodness-of-fit parameter $\chi^{2}$ as a function of $R_{c}$ for several sets of the cosmological parameters. As one can see, even adopting the most conservative case, $R_{c}$ larger than 2.0 is excluded at $95 \%$ C.L.

\subsection{General Case}

Now, we are ready to study the most general case, the case with $R \neq 0$ and $\alpha \neq 0$. In this case, the total angular power spectrum is written in the form

$$
C_{l}=C_{l}^{(\delta \chi)}+R^{2}\left[C_{l}^{(\delta \phi)}\right]_{R=1}+\alpha^{2}\left[C_{l}^{(\delta \chi)}\right]_{\alpha=1} .
$$

Effects of the non-vanishing $R$ - and $\alpha$-parameters can be read off from Fig. 3; normalizing the SW tail, non-vanishing $R_{b}$ increases the heights of the acoustic peaks while nonvanishing $R_{c}, \alpha_{b}$, and $\alpha_{c}$ suppress the acoustic peaks.

First, let us consider the baryonic isocurvature perturbations. In Figs. 11 and 12, we show the contours of the constant $C_{1 \mathrm{st}} / C_{10}$ and $C_{1 \mathrm{st}} / C_{2 \text { nd }}$ on the $R_{b}$ vs. $\alpha_{b}$ plane, where $C_{1 \mathrm{st}}$ and $C_{2 n d}$ are the heights of the first and second acoustic peaks, respectively. As one can see, larger value of $R_{b}\left(\alpha_{b}\right)$ corresponds to larger (smaller) ratio of $C_{1 \mathrm{st}} / C_{10}$. Importantly, the correlated and uncorrelated entropy perturbations affect the heights of the acoustic 


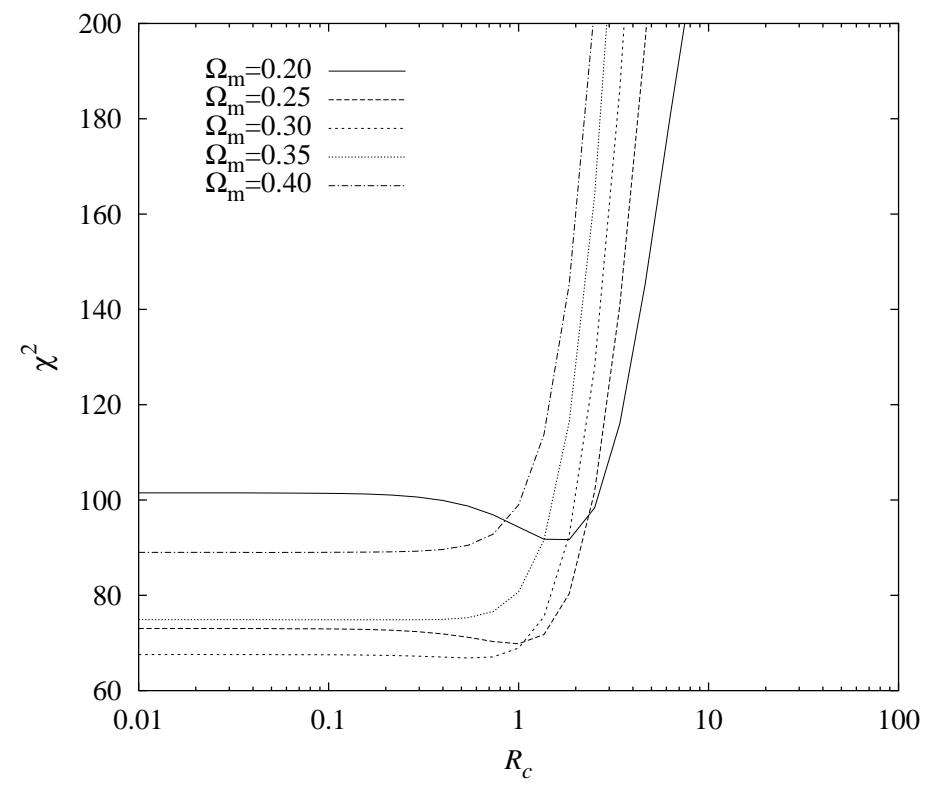

Figure 10: $\chi^{2}$ as a function of $R_{c}$. We take $\Omega_{b} h^{2}=0.019, h=0.65$, and the values of $\Omega_{m}$ are shown in the figure. The flat universe is assumed.

peaks in the opposite way. Thus, even with extremely large values of the $R_{b^{-}}$and $\alpha_{b^{-}}$ parameters, the ratio $C_{1 \mathrm{st}} / C_{10}$ may take a similar value as that from the adiabatic case if $R_{b}$ and $\alpha_{b}$ are properly related. The ratio $C_{1 \mathrm{st}} / C_{2 \text { nd }}$ is less sensitive to the parameters $R_{b}$ and $\alpha_{b}$, and for larger (smaller) value of $R_{b}\left(\alpha_{b}\right)$, the ratio $C_{1 \mathrm{st}} / C_{2 \text { nd }}$ is suppressed. We also calculated the ratio $C_{3 \text { rd }} / C_{2 \text { nd }}$ (with $C_{3 \text { rd }}$ being the height of the third acoustic peak) and found that this ratio is almost independent of $R_{b}$ and $\alpha_{b}$. This is because the structure of the angular power spectrum at high multipole is dominantly determined by the adiabatic part of the perturnations. We would like to emphasize that the effects of the primordial fluctuations of the $\phi$ and $\psi$ field are quite different; $C_{l}^{(\delta \phi)}$ is enhanced at high multipole while $C_{l}^{(\delta \psi)}$ becomes larger at low one. Thus, if the parameters $R_{b}$ and $\alpha_{b}$ obey a specific relation, the adiabatic result may be mimiced even when the effects of the entropy perturbation are quite large.

To study this issue, we calculate the goodness-of-fit parameter $\chi^{2}$ as a function of $R_{b}$ and $\alpha_{b}$ (with $R_{c}=\alpha_{c}=0$ ). Here, the overall normalization of $C_{l}$ is chosen such that the $\chi^{2}$ variable is minimized. The results are shown in Fig. 13 for several sets of the cosmological parameters. Here, all the primordial density perturbations are assumed to be scale-invariant. For the cases with $\Omega_{m}=0.2$ and 0.3 , it is interesting that large values of $R_{b}$ and $\alpha_{b}$ are allowed. In particular, for the case with $\Omega_{m}=0.2$, only the case with non-vanishing $R_{b}$ and $\alpha_{b}$ is allowed. Importantly, in this parameter region, the CMB anisotropy originates to non-adiabatic density perturbations while the contribution from the inflaton fluctuation $C_{l}^{(\delta \chi)}$ becomes negligibly small. In the following, we call this case 


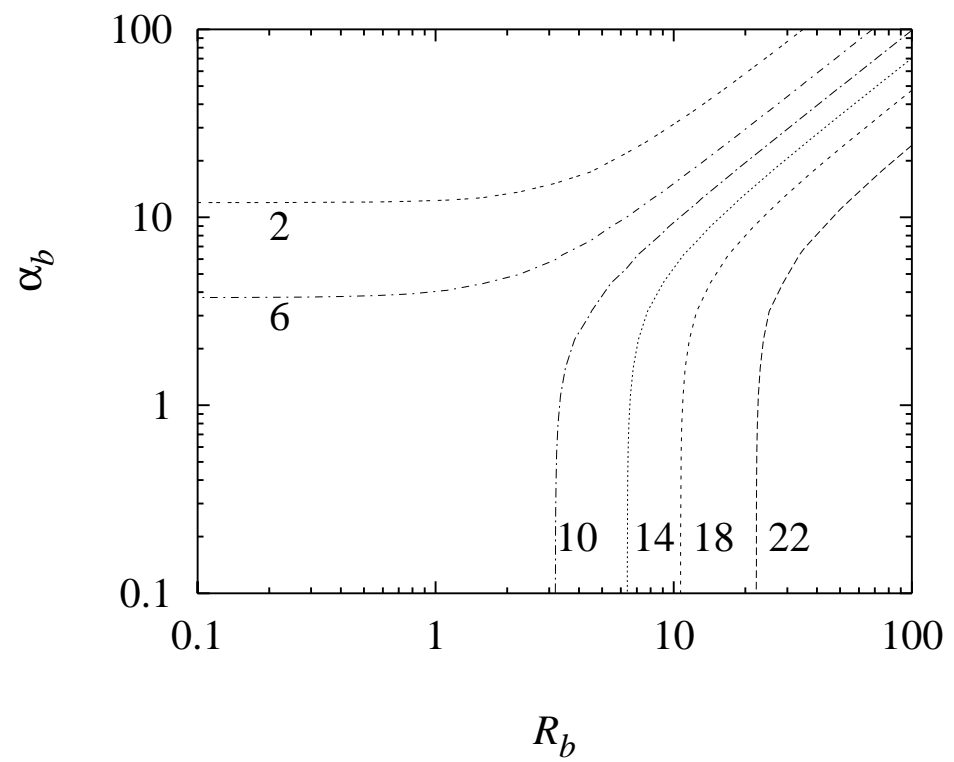

Figure 11: Contours of constant $C_{1 s t} / C_{10}$ on the $R_{b}$ vs. $\alpha_{b}$ plane. (The ratios are shown in the figure.) Here, $R_{c}=\alpha_{c}=0$ and we take the cosmological parameters same as Fig. 3.

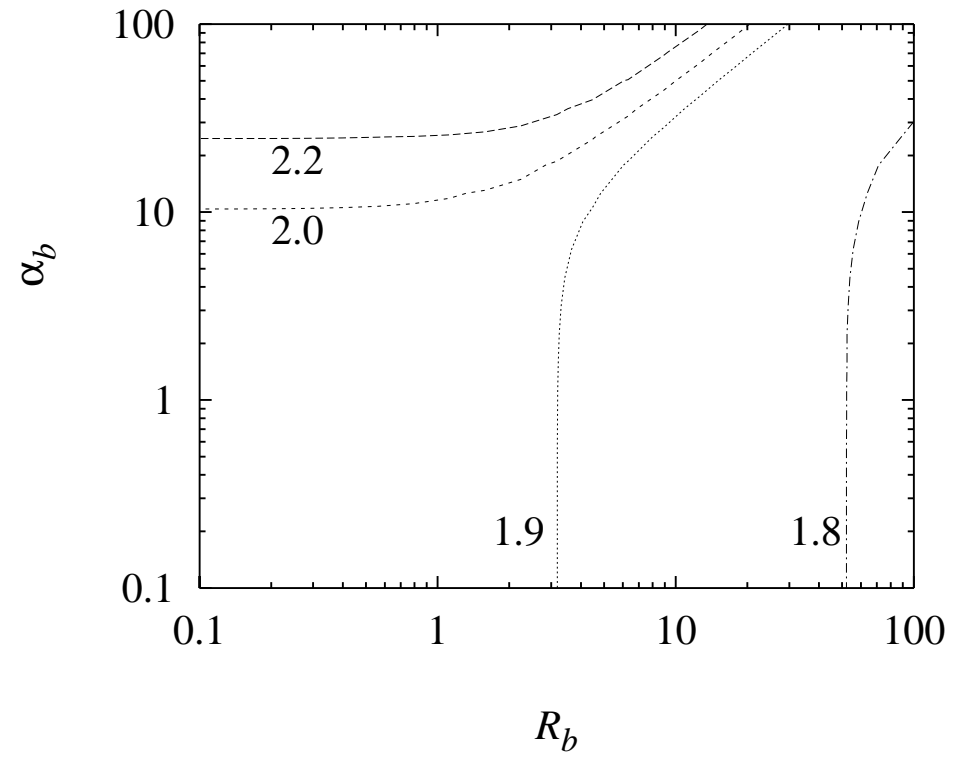

Figure 12: Same as Fig. 11, except that the contours are those of constant $C_{1 \mathrm{st}} / C_{2 \mathrm{nd}}$. 


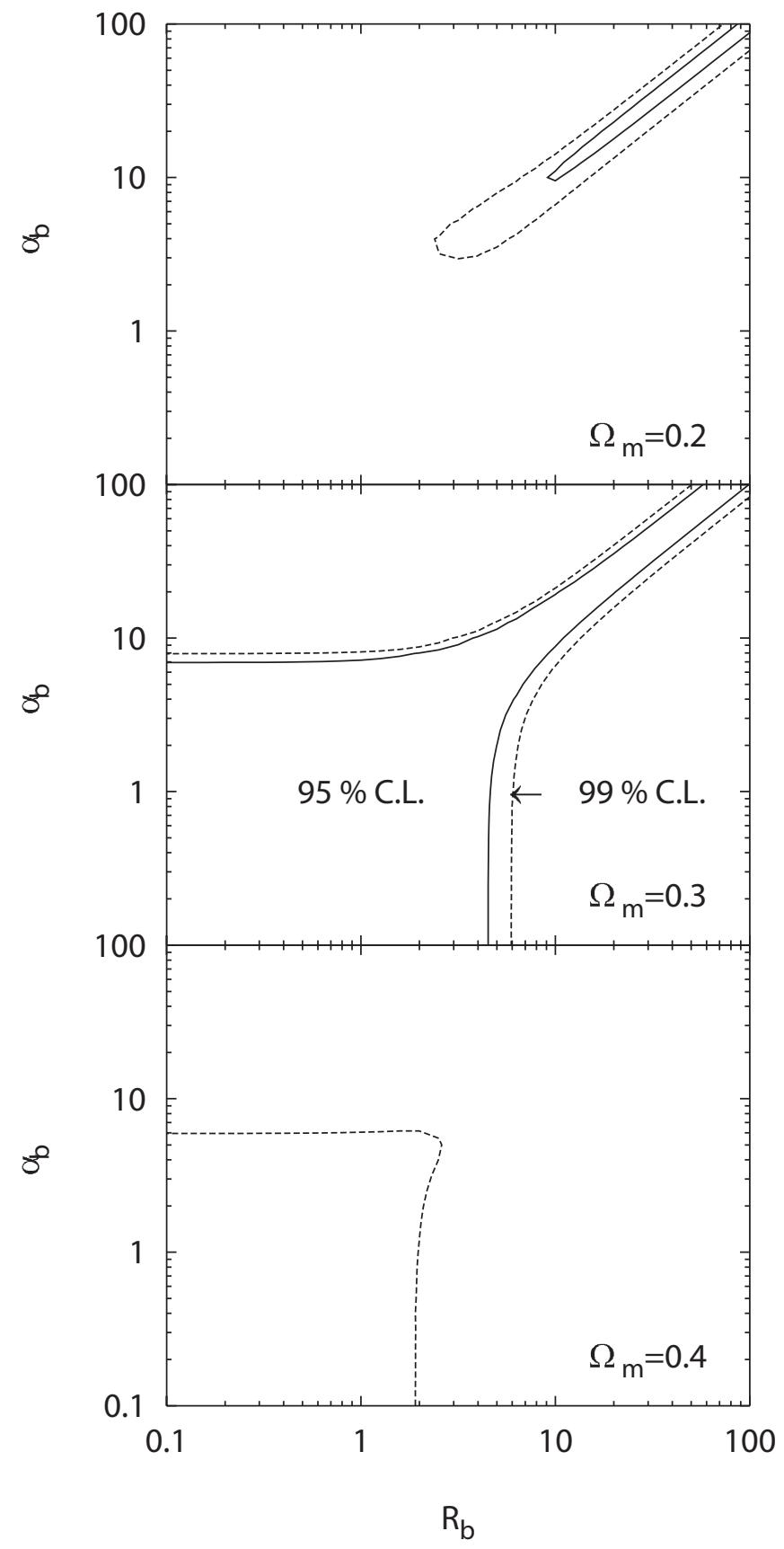

Figure 13: Contours of constant $\chi^{2}$ on the $R_{b}$ vs. $\alpha_{b}$ plane. We show contours of $\chi^{2}=84$ (solid line) and 93 (dashed line), which correspond to $95 \%$ and $99 \%$ C.L. allowed region with the $\chi^{2}$ statistics with 64 degrees of freedom, respectively. Here we take $\Omega_{b} h^{2}=0.019$, $h=0.65$, and the values of $\Omega_{m}$ are shown in the figures. 


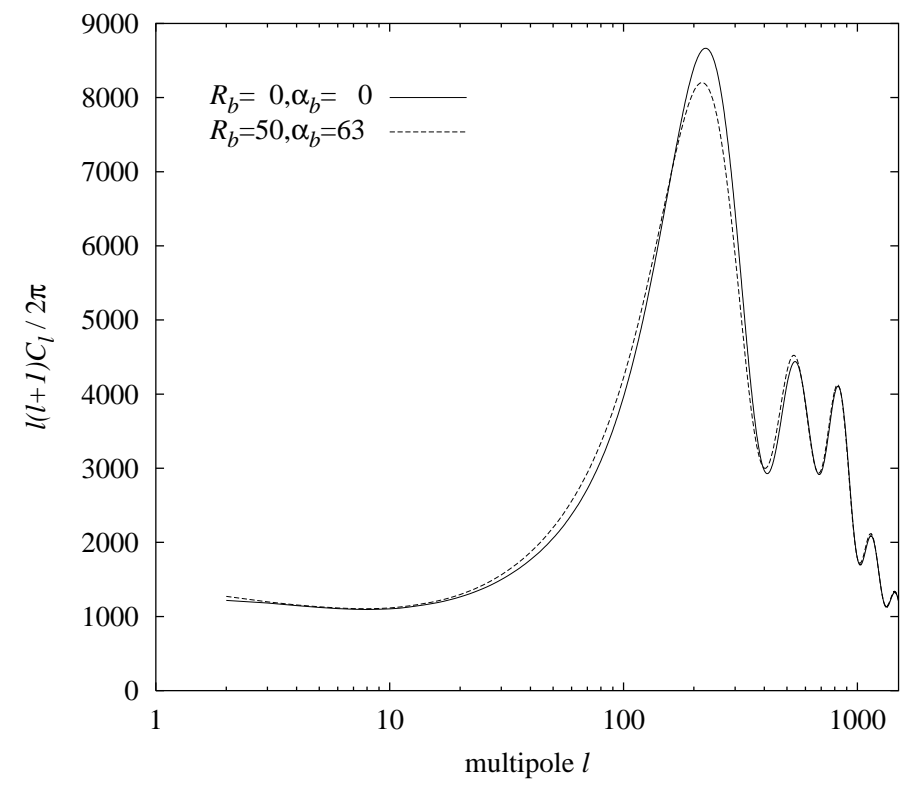

Figure 14: The angular power spectrum with $R_{b}=\alpha_{b}=0$ (solid line), and $R_{b}=50$ and $\alpha_{b}=63$ (dashed line). We take $R_{c}=\alpha_{c}=0$. The cosmological parameters are the same as those in Fig. 3. The overall normalizations are arbitrary.

as "modified-curvaton" case. It should be noticed that, in the modified-curvaton case, the parameters $R_{b}$ and $\alpha_{b}$ satisfy the relation $\alpha_{b} \simeq(1.0-1.7) R_{b}$ for $\Omega_{m}=0.3$. For $\Omega_{m}=0.4$, large values of $R_{b}$ and $\alpha_{b}$ are not allowed in contrast to other cases.

It is interesting to study whether the angular power spectrum in the modified-curvaton case can be distinguished from the adiabatic one by the on-going and future experiments. In Fig. 14, we plot the $\mathrm{CMB}$ angular power spectrum in the adiabatic and modifiedcurvaton cases, $R_{b}=\alpha_{b}=0$, and $R_{b}=50$ and $\alpha_{b}=63$ (which minimizes the $\chi^{2}$ variable for $R_{b}=50$ ), respectively. In the modified-curvaton case, shape of the power spectrum at high multipole is determined by $C_{l}^{(\delta \phi)}$ which is similar to that of the adiabatic case. Thus, it is difficult to distinguish two cases just by looking at the power spectrum at high multipole. However, as one can see, the structure of the angular power spectrum at relatively low multipole is significantly affected. In particular, the ratio $C_{1 \mathrm{st}} / C_{2 \text { nd }}$ will provide us an interesting probe for studying the modified-curvaton scenario. Since the MAP is expected to measure the angular power spectrum at $O(1 \%)$ level up to the multipole $l \sim 1000-2000, C_{l}$ at the high multipole may be used to calibrate the power spectrum to see the distortion of the CMB angular power spectrum from the adiabatic result.

We can also consider the case where the CDM sector has correlated and uncorrelated entropy perturbations (i.e., the case with $R_{c} \neq 0$ and $\alpha_{c} \neq 0$ ). Importantly, correlated and uncorrelated entropy perturbations both suppress the angular power spectrum at 
high multipole relative to the low one. Thus, in this case, too large $R_{c}$ is always excluded irrespective of the value of $\alpha_{c}$ if the baryon is from the decay product of $\phi$.

\section{Conclusion}

In this paper, we discussed the effects of the late-time entropy production due to the decay of the scalar-field condensations on the cosmic density perturbations. If the universe is reheated by the decay of the scalar field $\phi$, many of the components in the present universe are generated from the decay products of the $\phi$ field. In such a case, cosmic density perturbations are affected by the fluctuation of the amplitude of $\phi$ which may be generated during the inflation.

If all the components in the universe originate to the decay product of $\phi$, density perturbations generated from the primordial fluctuation of $\phi$ becomes adiabatic. In this case, the CMB angular power spectrum from the fluctuation of $\phi$ becomes the usual adiabatic ones with (almost) scale-invariant spectrum. If this becomes the dominant part of the cosmic density perturbations, then we have seen that the constraints on inflation models from observations of the CMB angular power spectrum are drastically relaxed.

If the baryon or the CDM is not generated from $\phi$ but from a new scalar field $\psi$, on the contrary, correlated mixture of the adiabatic and isocurvature perturbations may arise. In particular, if the $\psi$ field starts to oscillate much before the $\phi$ field dominates the universe, the metric and entropy perturbations obey the model-independent relation. In this case, the CMB angular power spectrum may be significantly affected and the shape of the resultant power spectrum depends on which component has the correlated isocurvature perturbation. If the baryonic component has the correlated isocurvature perturbation, the density perturbations after the decay of $\phi$ become those given in Eq. (3.17). In this case, $C_{l}$ at high multipole is more enhanced relative to that at low multipole. On the contrary, if the CDM is not generated from $\phi$, correlated isocurvature perturbation can be induced in the CDM component as given in Eq. (3.18). In this case, heights of the acoustic peaks become lower relative to the SW tail. If there exists an contamination of these component into the total angular power spectrum, $C_{l}$ differs from the adiabatic one. The important point is that, in both cases, too much correlated isocurvature perturbations become inconsistent with the present observation of the CMB power spectrum.

Even with the correlated isocurvature perturbation in the baryonic sector, heights of the acoustic peaks can be reduced by introducing uncorrelated isocurvature perturbation. This may happen, for example, if we take account of the primordial fluctuation of the scalar field $\psi$. (For example, this scalar field may be the Affleck-Dine field.) In particular, if the sizes of the correlated and uncorrelated entropy perturbations are properly chosen, the resultant $\mathrm{CMB}$ power spectrum becomes consistent with the present observations without the effect of the inflaton fluctuation. Even in this case, the angular power spectrum is not exactly the same as the conventional adiabatic one and the deviation from the adiabatic result may be observed at on-going and future experiments. 
In summary, if there exists correlated mixture of the adiabatic and isocurvature perturbations, the total CMB angular power spectrum can be distorted and it can be a signal

of the late-time entropy production due to the decay of the scalar field condensation. The on-going and future experiments may observe such a signal.

Acknowledgment: We acknowledge the use of CMBFAST [31] and RADPACK [32] packages for our numerical calculations. This work is supported by the Grant-in-Aid for Scientific Research from the Ministry of Education, Science, Sports, and Culture of Japan, No. 12047201 and No. 13740138.

\section{References}

[1] C. Bennett et al., Astrophys. J. Lett. 464 (1996) L1.

[2] C.B. Netterfield et al., astro-ph/0104460.

[3] A.T. Lee et al., astro-ph/0104459.

[4] N.W. Halverson et al., astro-ph/0104489.

[5] MAP webpage, http://map.gsfc.nasa.gov.

[6] PLANCK webpage, http://astro.estec.esa.nl/SA-general/Projects/Planck.

[7] A.H. Guth, Phys. Rev. D23 (1981) 347.

[8] See, for example, R. Liddle, hep-ph/0201264.

[9] I. Affleck and M. Dine, Nucl. Phys. B249 (1985) 361.

[10] See, for example, J. Polchinski, "String Theory" (1998, Cambridge University Press).

[11] G.D. Coughlan, W. Fischler, E.W. Kolb, S.Raby and G.G. Ross, Phys. Lett. B131 (1983) 59.

[12] T. Moroi, M. Yamaguchi and T. Yanagida, Phys. Lett. B342 (1995) 105; M. Kawasaki, T. Moroi and T. Yanagida, Phys. Lett. B370 (1996) 52; T. Moroi and L. Randall, Nucl. Phys. B570 (2000) 455.

[13] H. Murayama and T. Yanagida, Phys. Lett. B322 (1994) 349; K. Hamaguchi, H. Murayama and T. Yanagida, Phys. Rev. D65 (2002) 043512.

[14] R. Durrer, M. Gasperini, M. Sakellariadou and G. Veneziano, Phys. Rev. D59 (1999) 043511; R. Durrer, M. Gasperini, M. Sakellariadou and G. Veneziano, Phys. Lett. B436 (1998) 66; A. Melchiorri, F. Vernizzi, R. Durrer and G. Veneziano, Phys. Rev. Lett. 83 (1999) 4464. 
[15] G. Veneziano, Phys. Lett. B265 (1991) 287; M. Gasperini and G. Veneziano, Astropart. Phys. 1 (1992) 1; Phys. Rev. D50 (1994) 2519.

[16] J. Khoury, B.A. Ovrut, P.J. Steinhardt and N. Turok, Phys. Rev. D64 (2001) 123522; J. Khoury, B.A. Ovrut, N. Seiberg, P.J. Steinhardt and N. Turok, hepth/0108187; J. Khoury, B.A. Ovrut, P.J. Steinhardt and N. Turok, hep-th/0109050.

[17] S. Mollerach, Phys. Rev. D42 (1990) 313.

[18] K. Enqvist and M.S. Sloth, Nucl. Phys. B626 (2002) 395.

[19] D.H. Lyth and D. Wands, Phys. Lett. B524 (2002) 5.

[20] T. Moroi and T. Takahashi, Phys. Lett. B522 (2001) 215.

[21] D. Langlois. Phys. Rev. D59 (1999) 123512; C. Gordon, D. Wands, B.A. Bassett and R. Maartens, Phys. Rev. D63 (2001) 023506; N. Bartolo, S. Matarrese and A. Riotto, Phys. Rev. D64 (2001) 083514; Phys. Rev. D64 (2001) 123504; L. Amendola, C. Gordon, D. Wands and M. Sasaki, astro-ph/0107089.

[22] M. Dine, L.J. Randall and S. Thomas, Phys. Rev. Lett. 75 (1995) 398; Nucl. Phys. B458 (1996) 291.

[23] W. Hu, Ph.D thesis astro-ph/9508126).

[24] J. Primack, astro-ph/0007187.

[25] J.R. Bond, A.H. Jaffe and L. Knox, Astrophys. J. 533 (2000) 19.

[26] J.M. Bardeen, P.J. Steinhardt and M.S. Turner, Phys. Rev. D28 (1983) 629.

[27] X. Wang, M. Tegmark and M. Zaldarriaga, astro-ph/0105091.

[28] N. Bartolo and A.R. Liddle, astro-ph/0203076; R. Durrer and F. Vernizzi, hepph/0203275; A. Notari and A. Riotto, hep-th/0205019; D.H. Lyth, hep-ph/0205266.

[29] D. Langlois and A. Riazuelo, Phys. Rev. D62 (2000) 043504.

[30] R. Stompor, A.J. Banday and K.M. Gorski, Astrophys. J. 463(1996) 8; S.D. Burns, astro-ph/9711303; T. Kanazawa, M. Kawasaki, N. Sugiyama and T. Yanagida, Prog. Theor. Phys. 102 (1999) 71; K. Enqvist, H. Kurki-Suonio and J. Valiviita, Phys. Rev. D62 (2000) 03003; Phys. Rev. D65 (2002) 043002.

[31] M. Zaldarriaga and U. Seljak, Astrophys. J. 469 (1996) 437.

[32] RADPACK webpage, http://flight.uchicago.edu/knox/radpack.html. 